\title{
Review Paper \\ Barriers and Facilitators of the Outpatient Health Service Use by the Elderly
}

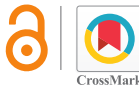

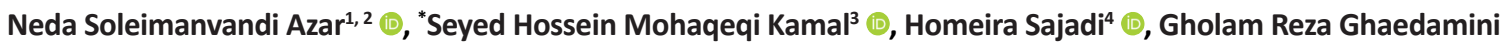

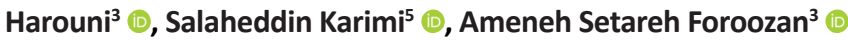

1.Preventive Medicine and Public Health Research Center, Psychosocial Health Research Institute, Iran University of Medical Sciences, Tehran, Iran.

2. Department of Social Welfare Management, School of Education Sciences and Social Welfare, University of Social Welfare and Rehabilitation Sciences, Tehran, Iran.

3. Social Welfare Management Research Center, University of Social Welfare and Rehabilitation Sciences, Tehran, Iran.

4. Social Determinants of Health Research Center, University of Social Welfare and Rehabilitation Sciences, Tehran, Iran.

5. Social Determinants of Health Research Center, Health Management and Safety Promotion Research Institute, Tabriz University of Medical Sciences, Tabriz, Iran

\begin{tabular}{|c|c|}
\hline $\begin{array}{l}\text { Use your device to scan } \\
\text { and read the article online }\end{array}$ & citation: Soleimanvandi Azar N, Mohaqeqi Kamal SH, Sajadi H, Ghaedamini Harouni GR, Karimi S, Foroozan AS. [Barriers \\
\hline 口ifing & $\begin{array}{l}\text { and Facilitators of the Outpatient Health Service Use by the Elderly (Persian)]. Iranian Journal of Ageing. 2020; 15(3):258-277. } \\
\text { https://doi.org/10.32598/sija.15.3.551.3 }\end{array}$ \\
\hline 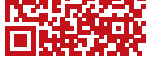 & dol' https://doi.org/10.32598/sija.15.3.551.3 \\
\hline
\end{tabular}

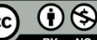

Received: 08 Feb 2020 Accepted: 10 May 2020 Available Online: 01 Oct 2020

Keywords:

Older adults, Health service use, Outpatient, Systematic reviews, Andersen's Behavioral Model of Health Service Use

\section{A B S TRACT}

Objectives Increasing care needs for the elderly are an important concern for different countries, especially those with an aging population. It is important for health policy making to have knowledge of the factors affecting the use of health services in the elderly to identify the potential problems and develop appropriate interventions for improving utilization and increasing access to health services. This study aims to investigate the barriers and facilitators of the outpatient health service use in the elderly Methods \& Materials In this systematic review, studies in English published from 1996 to 2019 were searched in Web of Science, PubMed and Scopus databases using PRISMA guidelines and related keywords. After eliminating duplicate and irrelevant articles, the quality of remaining articles was evaluated by two evaluators independently, based on STROBE checklist. Narrative synthesis method was used to combine the data

Results Forty-four eligible studies were included for the review. The determinants of the health service use were divided into three categories of predisposing factors (e.g. age, gender, marital status, ethnicity), enabling factors (e.g. income, insurance coverage, education level, employment status, social network, social support), and need factors (e.g. having chronic disease, self-assessed health status, severity of disease, number of diseases, comorbid diseases, physical disability, unhealthy lifestyle). Findings showed that age $>80$ years, ethnic minority, being unemployed and retired, low educational level, small and limited social network, and physical disability were the barriers to using outpatient health services, while being female, married, having insurance, social support, having a companion during a disease, having children, high income level, and shorter distance to the health care centers were the facilitators of using outpatient health services in the elderly

Conclusion A group of factors are associated with the outpatient health service use by the elderly. These factors include predisposing, enabling, and need-related factors according to Andersen's behavioral model of health service use. Interventions to increase the use of health services by the elderly should be based on these factors, and should be taken into account by the policymakers to reduce the burden of health services caused by diseases

\section{* Corresponding Author: \\ Seyed Hossein Mohaqeqi Kamal, PhD.}

Address: Social Welfare Management Research Center, University of Social Welfare and Rehabilitation Sciences, Tehran, Iran.

Tel: +98 (21) 71732860

E-mail: hosseinmohaqeq@gmail.com 


\section{Extended Abstract}

\section{Introduction}

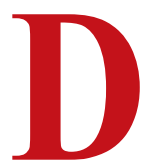

emographic changes, along with changes in the epidemiological pattern of diseases in old age, as well as limited human and financial resources, especially in developing countries, have made the provision of health facilities and services to the elderly face serious problems [18]. Rising care needs for the elderly are a concern of health care systems in countries with an aging population $[16,17]$. Given the challenges of aging population and the relatively high cost of health services for them, identifying the factors affecting the use of health services by the elderly is of particular importance for health decision-makers to determine potential problems and develop appropriate interventions for increasing access and remove barriers to health services [16, 27]. Therefore, the aim of this review study is to investigate the determinants, barriers, and facilitators of using outpatient health services for older people.

\section{Methods \& Materials}

In this systematic review, the search was conducted in Web of Science, PubMed, and Scopus databases among the studies in English from 1996 to 2019 according to PRISMA checklist and using following keywords: Health service Utilities, aging, older people, older adult, elderly, aged, factor, determinant, predictor, health care Utilities, health service use, health care use. Quantitative, observational, and cross-sectional studies using secondary and longitudinal analysis of factors affecting the use of outpatient health services by the elderly aged $\geq 60$ years were included in the study. Studies on the use of unofficial health services (e.g. from friends/ family, or religious support), studies with the immigrant, incarcerated, hospitalized elderly population, residents of nursing homes, and the elderly with certain illnesses including oral disease, mental illness, and cancers or disability were excluded. Studies that used data from health centers, as well as dissertations, review studies, and letters to the editors were also excluded. The search in databases yielded 300 articles. In addition, 3 new articles were found through search in the reference lists of articles. After removing duplicate and irrelevant articles, 47 articles remained and entered the qualitative evaluation phase. The quality of the articles was evaluated independently by two raters based on the STROBE checklist. After quality assessment, finally 44 articles entered into the review. In order to combine the data of these 44 articles, the narrative synthesis method was used.

\section{Results}

In this study, the quality of 44 articles was confirmed. Andersen's behavioral model of health service utilization was used as a theoretical framework to classify the Results of this study into three categories: Predisposing factors (Age, gender, marital status, ethnicity), Enabling factors (Education level, employment status, income level, insurance coverage, social network, social support, place of residence) and Need factors (Having a chronic disease, self-assessed health status, need for health care services, severity of the disease, number of diseases, comorbid diseases, type and duration of disease, having disability or functional limitation, unhealthy lifestyle). Findings of the reviewed articles showed that with the increase of age, the need to use health services increases $[3,15,25,33,37,39,40,43,50,53$, $54,56,59]$, but in some studies, with increasing age and exceeding 85 years, the use of health services decreased $[55,58,61]$. Gender was associated with access to health services such that women were significantly more likely to seek health services $[5,9,15,17,25,30,39,43,45,49$, 50, 52, 53, 54, 58, 59, 61, 64]. Married people used more health services than divorced, single, and never married people $[25,30,43,52,57,60]$.

Ethnic minorities had less access to health services than the dominant ethnic group [63]. As income increased, the use of health services increased and vice versa. This relationship was also true based on satisfaction with economic status, household assets, individual wealth, household expenses, monthly and annual household income $[5,17,25$, 30, 31, 37, 39, 43, 47, 52, 53, 58, 59, 61]. Regarding employment status, retired and unemployed elderly people used less health services than employed elderly people $[3,12,64]$. Several studies have linked higher education to health service use $[1,3,25,35,43,47,52]$, although some studies showed that this linear relationship is not always established and higher education was related to using less outpatient health services in the elderly $[5,16,17,37$, 58, 61]. Having health insurance, including commercial and private insurance, and the amount of costs covered by insurance was associated with greater access to health services in the elderly $[25,41,43,47,59,63,64]$. Wider social network [61], family social support [46], having a caregiver or a companion during disease, and having a child [46] were associated with more access to health services, and in various studies living alone was one of the causes of lack of use [33, 49].

Studies showed that rural life is not only associated with low health service use $[5,25,31,35,43,47,54]$; in studies by Park (2018) and Pletzer (2014), elderly people living in rural areas used outpatient health services more than the 
Table 1. Factors affecting the use of outpatient health services by the elderly according to the reviewed articles

\begin{tabular}{|c|c|}
\hline Factors & Number of Related Studies \\
\hline Predisposing & No. \\
\hline Gender $[5,9,15,17,25,30,31,39,43,45,49,50,52-54,58,59,61,64]$ & 19 \\
\hline Age $[3,15,25,33,37,39,40,43,50,53-56,58,59,61]$ & 16 \\
\hline Marital status $[5,25,30,43,52,57,60,63]$ & 8 \\
\hline Ethnicity $[37,63]$ & 2 \\
\hline Enabling & - \\
\hline Income $[3,5,9,15,17,30,43,49,50,52,59,64,32,37,47,53,62]$ & 17 \\
\hline Insurance coverage $[25,41,43,47,59,63,64]$ & 7 \\
\hline Socio-economic status $[3,5,17,31,37,39,47,52,53,58,59,62]$ & 12 \\
\hline Education level $[1,5,3,25,35,43,47,52,16,17,37,58,61]$ & 13 \\
\hline Employment status $[3,12,64]$ & 3 \\
\hline Place of residence $[5,25,31,35,43,47,54,39,61]$ & 10 \\
\hline Social network [61] & 1 \\
\hline Social support [46] & 1 \\
\hline Living alone $[33,49]$ & 2 \\
\hline Distance to health care centers $[3,46]$ & 2 \\
\hline Having a caregiver or companion during disease [46] & 1 \\
\hline Having a child [46] & 1 \\
\hline High living standards [46] & 1 \\
\hline Health knowledge and information [48] & 1 \\
\hline Need & - \\
\hline Self-assessed health status $[3,5,9,12,15,31,50,52,53,59]$ & 10 \\
\hline Type of disease (acute or chronic) / duration of the disease $[1,5,9,12,16,17,25,40,42,43,47,49,52-55,57,59]$ & 18 \\
\hline Low mental health and cognitive impairment $[38,46,49,50,57,61]$ & 6 \\
\hline Physical disability and functional limitation [25] & 1 \\
\hline Feeling the need for health care services $[15,58]$ & 2 \\
\hline Having comorbid diseases [55] & 1 \\
\hline Physical activity $[12,51,59,36]$ & 4 \\
\hline Feeling pain $[25,46]$ & 2 \\
\hline Correct perception of the problem [46] & 1 \\
\hline Unhealthy lifestyle (smoking and alcohol consumption) [25] & 1 \\
\hline
\end{tabular}


elderly living in urban areas $[54,61]$. Shorter duration or distance in access to health care centers increased the likelihood of using health services in the elderly $[3,46]$. Having chronic diseases $[1,5,9,12,16,17,25,40,42,43,47$, $49,52-55,57,59]$, low mental health, depression [38, 46, $49,50,57,61]$, cognitive impairment [61], physical disability [25], low self-assessed health status [3, 5, 9, 12, 15, $31,50,52,53,59]$, correct perception of the problem [46], need for health care services $[15,58]$, comorbid diseases [55], feeling pain $[25,46]$ and unhealthy lifestyle (smoking and alcohol consumption) [25] were associated with using more outpatient health services (Table 1).

\section{Conclusion}

A set of factors are consistently associated with the use of health services by the elderly. The present study identified the factors affecting the use of outpatient health services by the elderly according to Andersen's behavioral model of health service use. It seems that interventions to increase the use of health services in the elderly should be based on three categories of predisposing, enabling and need-related factors. Given that most of the reviewed studies indicated to the variables of income, insurance coverage, age $>80$ years, and having chronic diseases, these factors are then the most important factors affecting the use of health services in the elderly which should be considered as the policy makers' health policy priorities for the elderly so as not to increase the burden of services caused by diseases.

\section{Ethical Considerations}

\section{Compliance with ethical guidelines}

The present study obtained its ethical approval from the University of Social Welfare and Rehabilitation Sciences (Code: IR.USWR.REC.1397.029).

\section{Funding}

The project was funded by the Deputy of Social and Cultural Affairs of Tehran Municipality (Grant number: 990.473667). Also, This review study was part of a PhD. dissertation of second author at Department of Social Welfare Management, School of Education Sciences and Social Welfare, University of Social Welfare and Rehabilitation Sciences.

\section{Authors' contributions}

All authors had equal contribution in preparing this article.

\section{Conflicts of interest}

The authors declare no conflict of interest

\section{Acknowledgements}

We thank Professor Hossein Malek Afzali for his guidance and scientific support. 
This Page Intentionally Left Blank 


\title{
موانع و تسهيل كنندهاي بهرهمندى از خدمات سلامت سريايى در سالمندان: يك مرور نظاممند
}

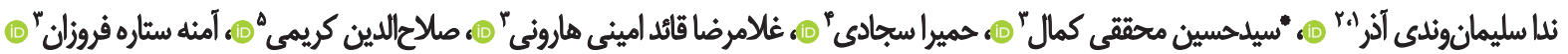

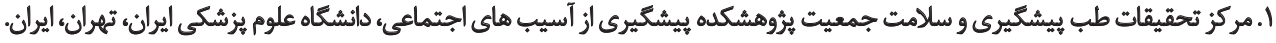

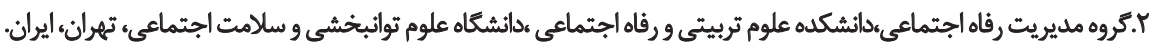

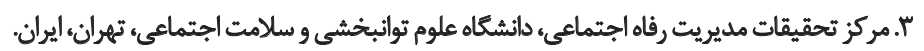

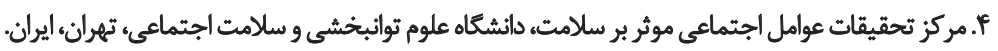

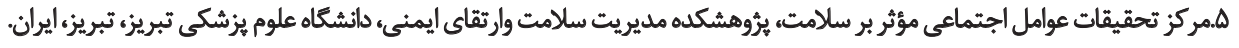

\section{حكبن}

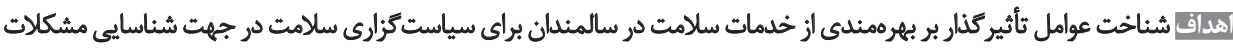

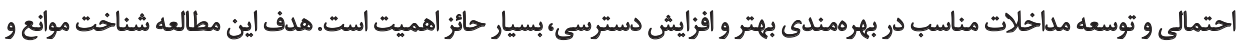

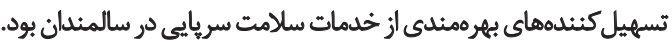

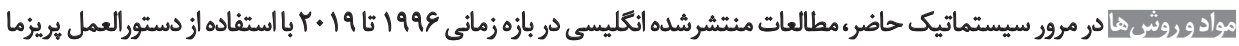
وكلمات كليدى مرتبط، به صورت الكتروئيكى از بايكاهماي اطلاعاتئى

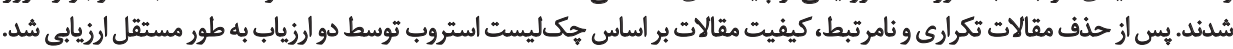

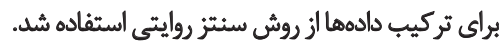

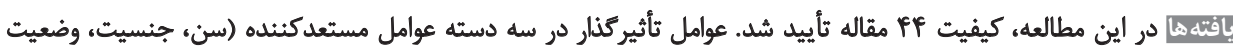

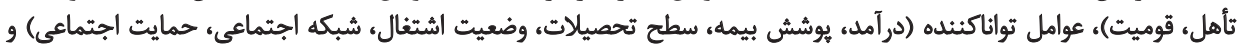

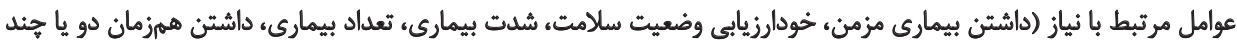

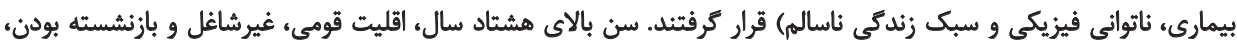

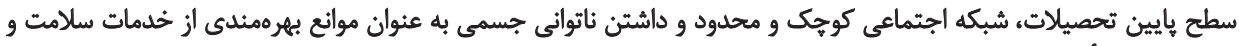

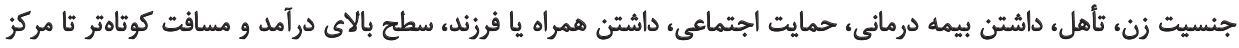

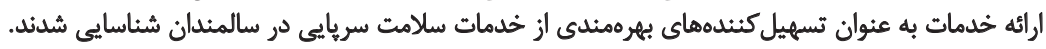

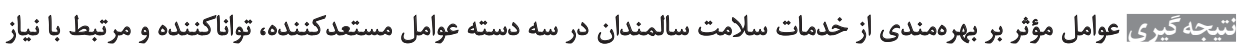

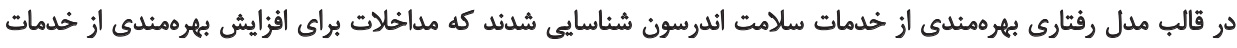

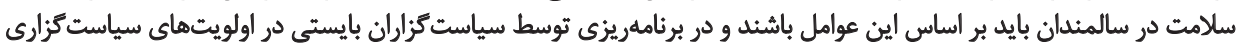

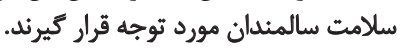

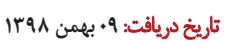

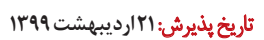 \\ تاريخ انتشار: 11 تير
}

سالمندة بهمرهمئدى

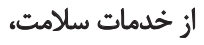

سريايیى، مرور سيستماتيك، مدل مرول رفتارى اندريسون

جمعيت سالمندان جهان به سرعت در حال رشد است و در جهان

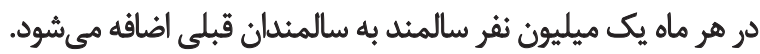

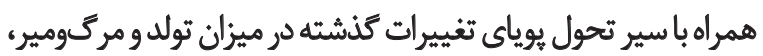

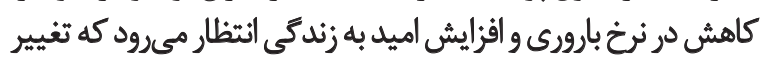

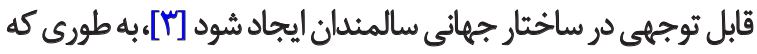

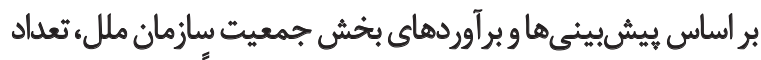

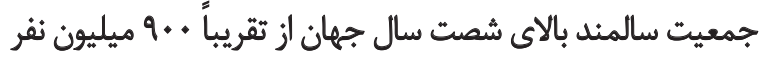

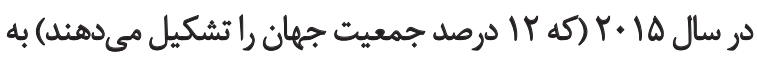

سالمندى جمعيت توسط سازمان ملل به عنوان يكى از بارزترين

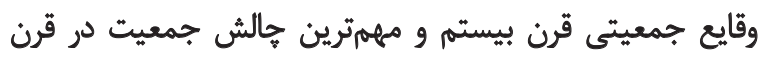

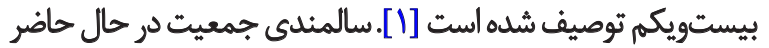

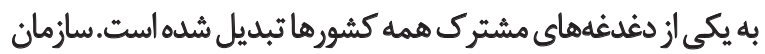

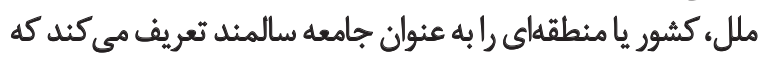

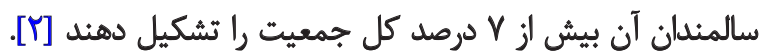

$$
\begin{aligned}
& \text { : نويسئده مسئول: } \\
& \text { دكتر سيد حسين محققى كمال }
\end{aligned}
$$

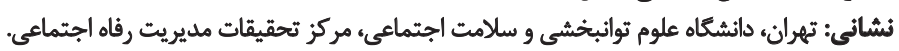

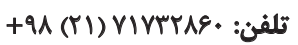

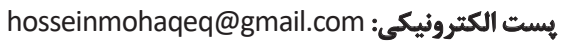


مدل رفتارى بهرهمندى از خدمات سلامت رونالد ام. اندرسون'

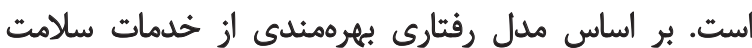

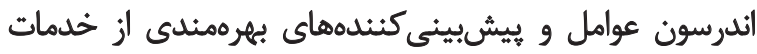

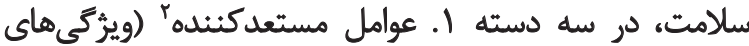

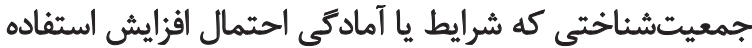

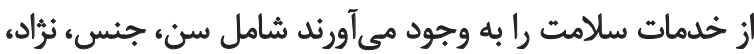

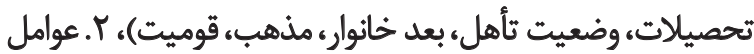

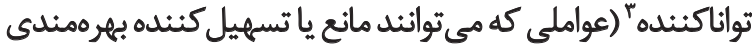

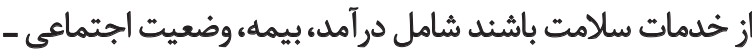

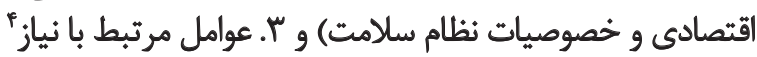

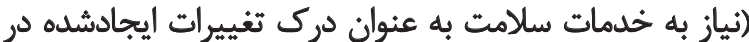

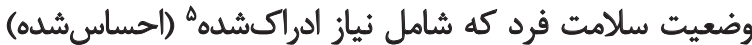

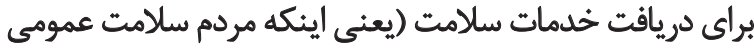

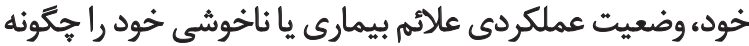

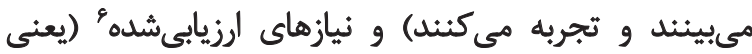

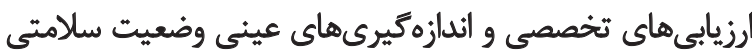

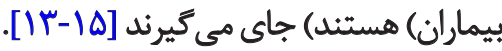

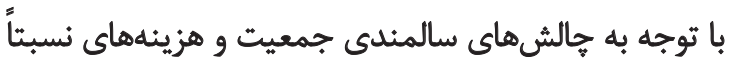

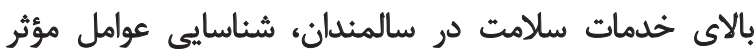

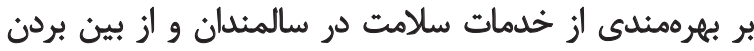

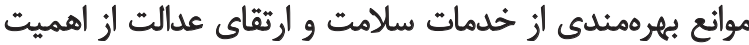

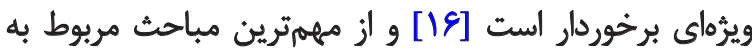

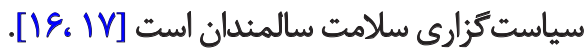

تغييرات جمعيتشناختى به همراه تغيير الكوى هملكيرشناختي

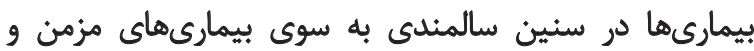

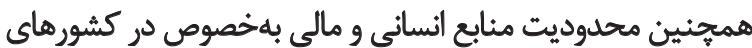

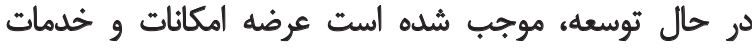

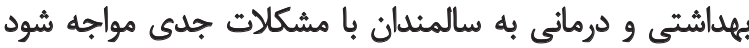

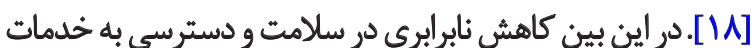

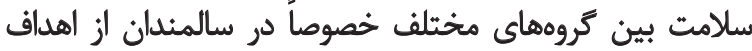

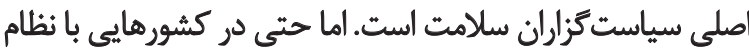

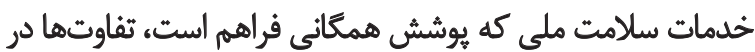

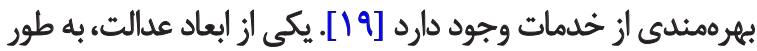

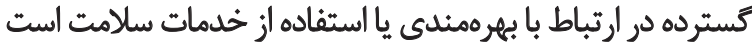

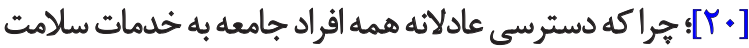

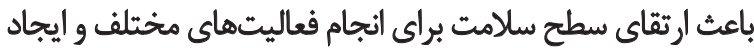

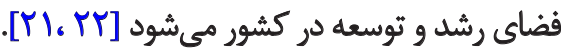

1. Andersen's Behavioral Model of Health Service Utilization

2. Predisposing factor

3. Enabling factor

4. Needs factor

5. Perceived needs

6. Evaluated needs

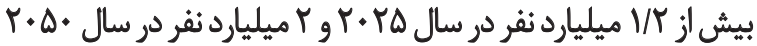

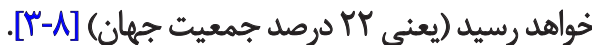

افزايش جمعيت سالمندان به طور اجتنابنايذيرى باعث

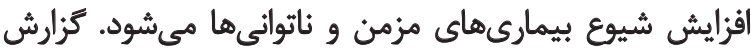

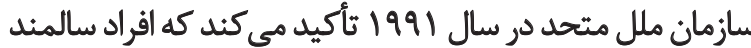

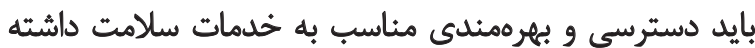

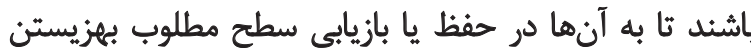

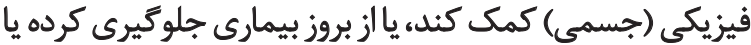

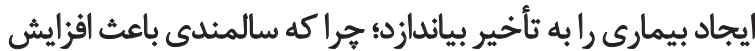

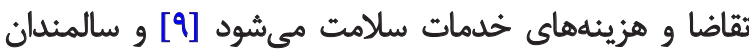
بزركترين كروه مصرف كنيده (استفاده كنينده) از خدمات سلات سلامت

در اكثر كشورهاى صنعتى و در حال توسعه هستند [ـ • [1] شيوع بالاى بيمارىهاى مزمن و عوارض ناتوان كنينده آن در

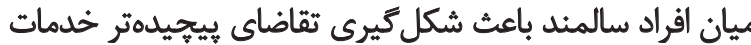

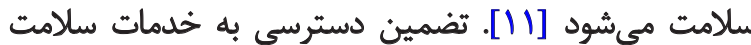

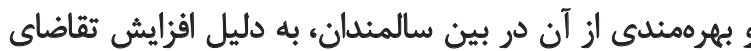

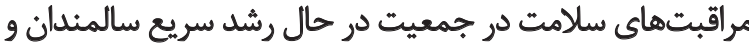

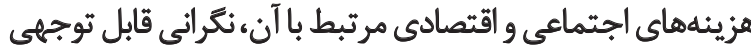
در سراسر جهان ايجاد كرده است [11].

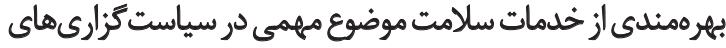

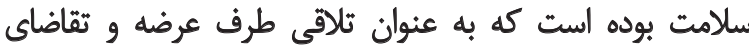

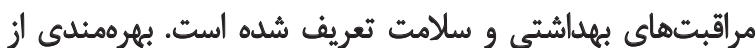

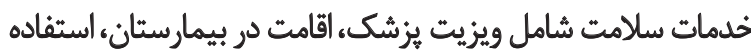

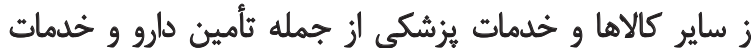

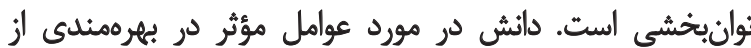

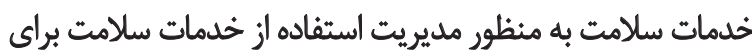

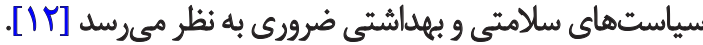
تصميم فرد براى استفاده از خدمات سلامت نتيجه تعامل

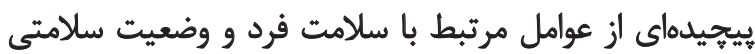

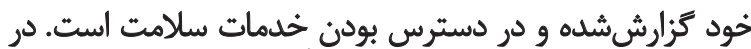

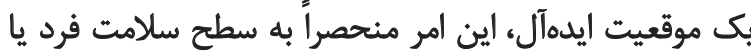

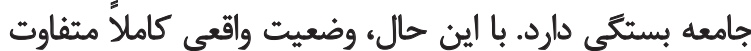

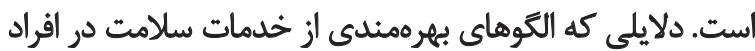

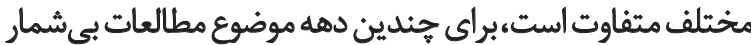

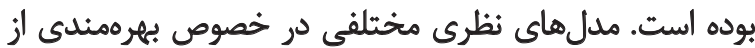

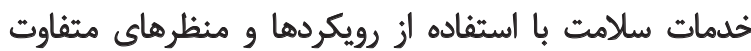

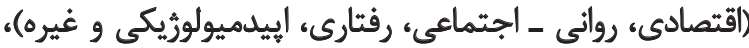

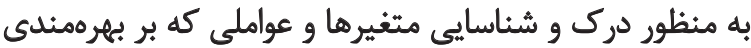

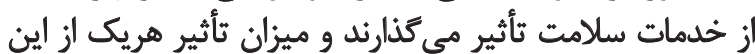

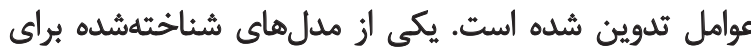

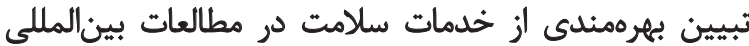


سيتامبر 19 1 انجام شد.

استراتثى جستوجو

جستوجوى نظاممند براى شناسايى مقالات منتشرشده

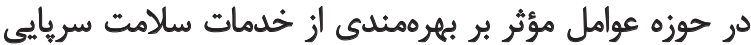

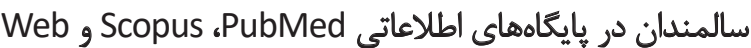
of Science Health service utili*, Aging, كليدى بهكار كلئه شام Older people, Older adult, Elder*, Aged, Factor, Determinant, Predictor, Health care utili*, Health service use, Health care use كليدى در Medical Subject Headings (MeSH) انجام شد. همجينين جستوجوى دستى فهرست منابع مطالعات منتشرشده نيز صورت كرفت. كلمات كليدى و تركيبات آنها بر بر اساست

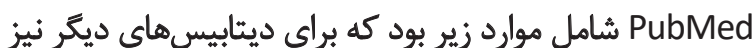
معادل مازى شد.

(Aged [Title/Abstract] OR Elder* [Title/Abstract] OR "Older Adults" [Title/Abstract] OR "Older People" [Title/Abstract] OR Aging [Title/Abstract] OR Geriatric [Title/Abstract]) AND ("Health service utilization" [Title] OR "Health care utilization" [Title] OR "Health care utili*" [Title] OR "Health service utili*" [Title] OR "Health service use" [Title] OR "Health care use" [Title]) AND (Determinant [Title/Abstract] OR Factor [Title/Abstract] OR Predictor [Title/Abstract])

\section{معيارهاي ورود وخروج}

مطالعات كمى، از نوع مشاهدهاى، مقطعى، تحليل ثانويه و بر باني

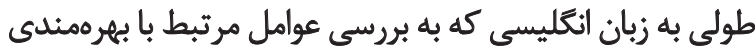

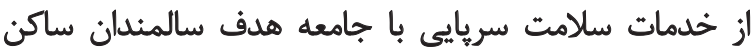

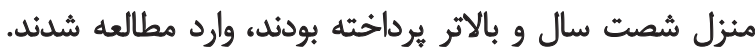

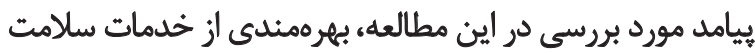

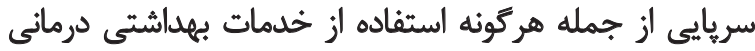

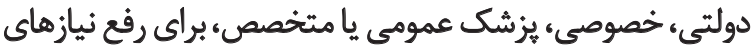

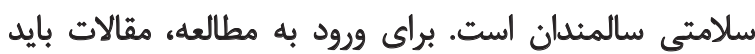

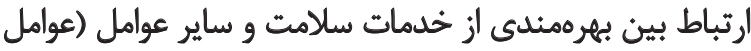

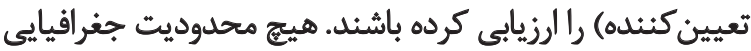

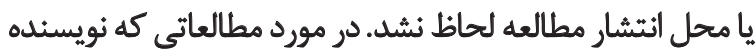

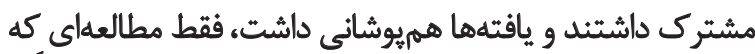

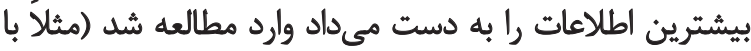

8. Preferred Reporting Items for Systematic Reviews and MetaAnalyses (PRISMA)
يكى از اهداف تعيين تقاضا در بخش بهداشت و درمان، تعيين

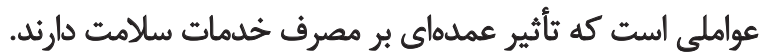

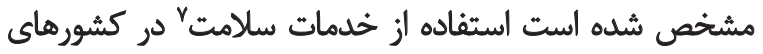

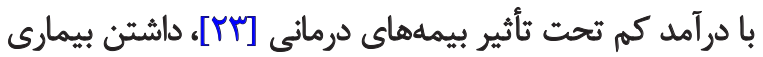

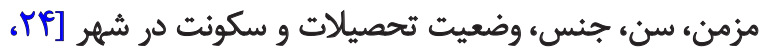

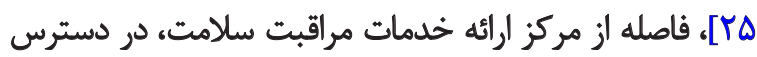

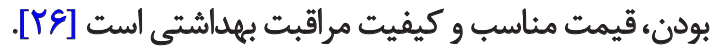

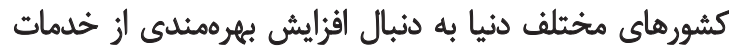

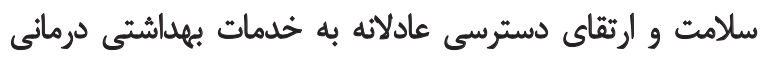

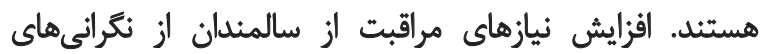

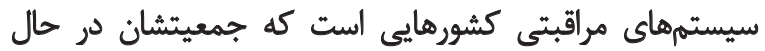

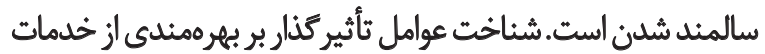

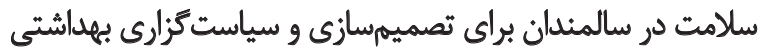

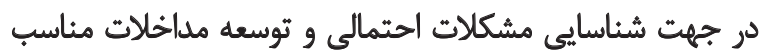

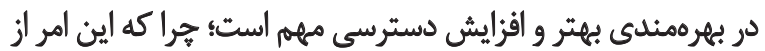

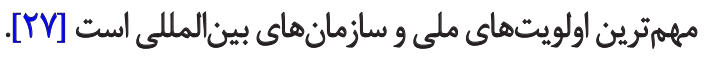
تحقيقات مرتبط با بهرهمندى از خدمات سلامت، به ما اجازه

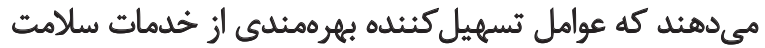

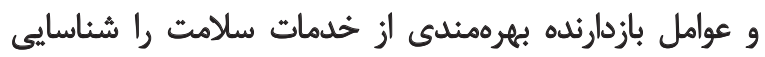

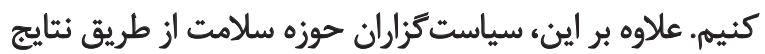

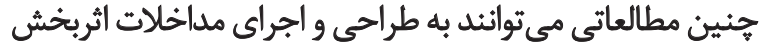

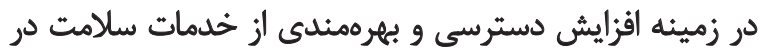

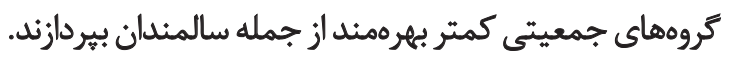
باتوجه به اينكه بهرهمندى از خدمات سلامت در توسعه جامعه

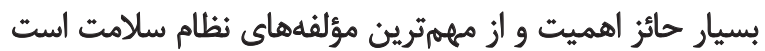

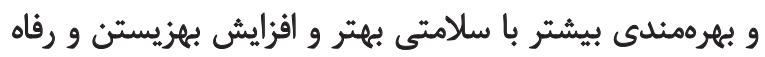

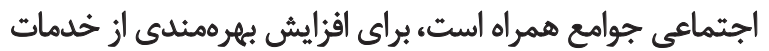

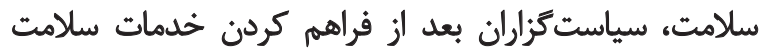

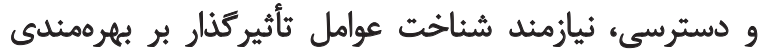

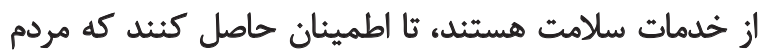

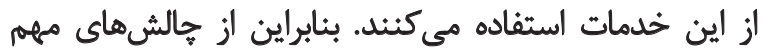

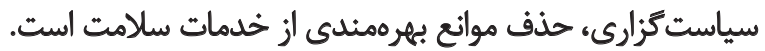

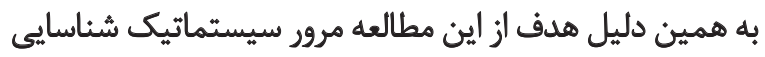

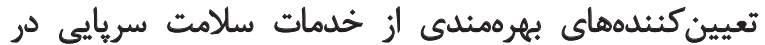

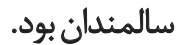

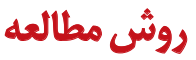

مطالعه حاضر يك مرور سيستماتيك روى كليه مطالعات

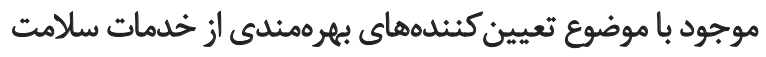

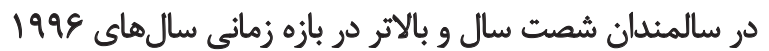

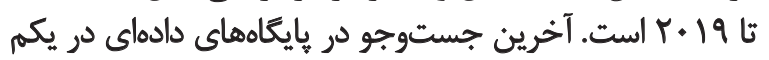

7. Health service utilization 


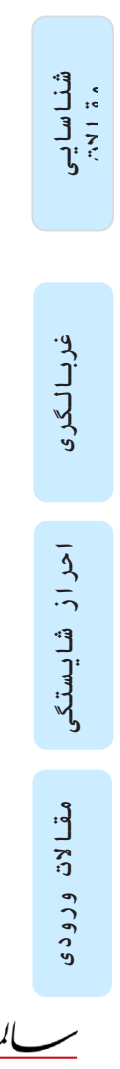

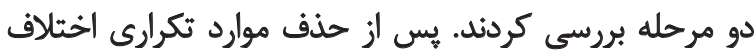

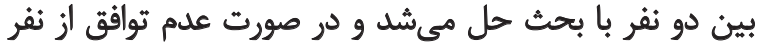

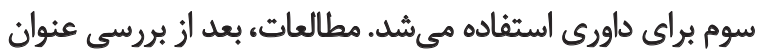

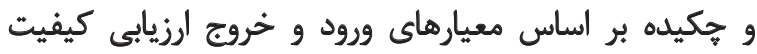

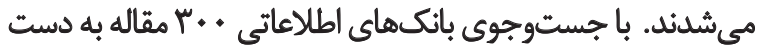

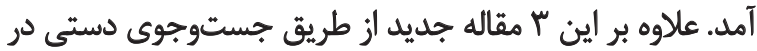

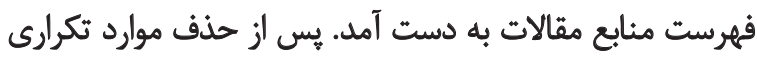

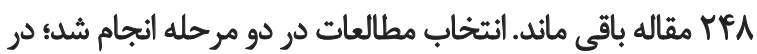

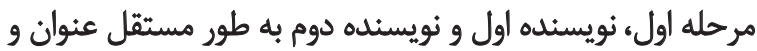

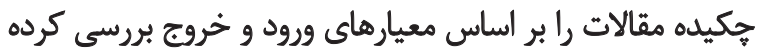

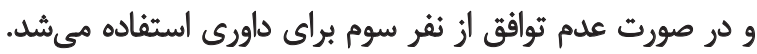

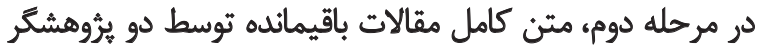

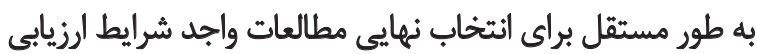

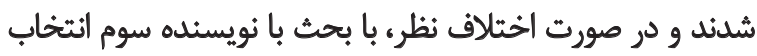

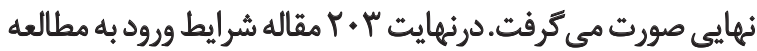
را نداشته و FV مقاله وارد فاز ارزيابى كيفى شدند.

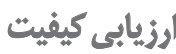

كيفيت مطالعات انتخابشده توسط دو نفر و با استفاده از

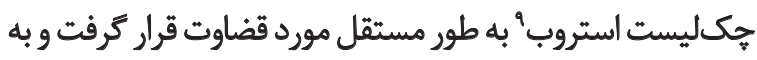

9. STROBE (Strengthening the Reporting of Observational Studies in Epidemiology)
تصوير 1. مقالات شناسايىشده در مراحل غربالكرى، احراز شرايط و ورود به مطالعه

$$
\text { حجم نمونه بيشتر، تحليل هاى قابل اعتمادتر). }
$$

مطالعات انجامشده در مورد استفاده از خدمات سلامت

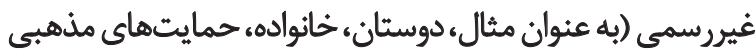

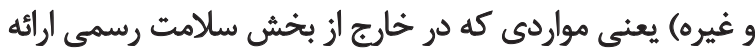

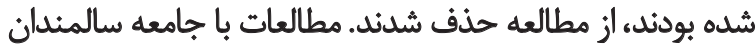

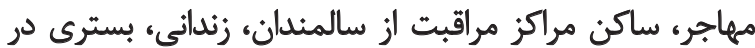

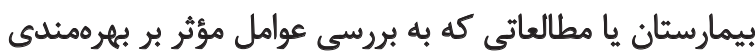

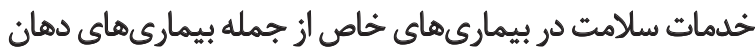

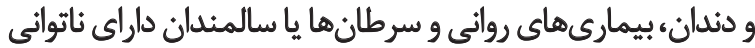

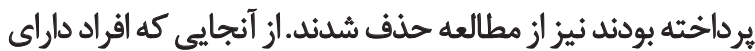

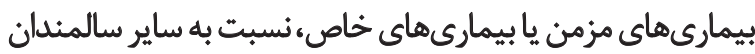

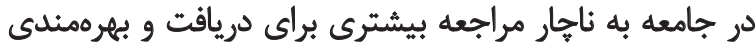

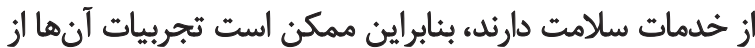

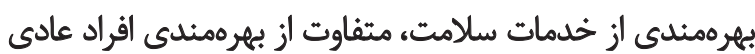

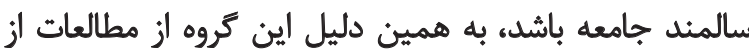

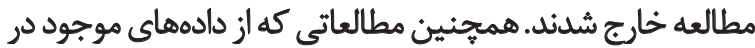

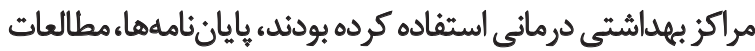
مرورى و نامه به سردبير نيز حذف شدئد.

$$
\text { معيار هاي انتخاب }
$$

دو نفر مروركر به طور مستقل مطالعات بهدستآمده از بانكهاي اطلاعاتى را بر اساس عنوان، جهكيده و متن كامل در در 
جدول ا. ويرٔكى هاى مطالعات مرورشده

\begin{tabular}{|c|c|c|c|c|c|c|c|}
\hline امثيفز ارزيابى & ميحل انجام مطالعه & حجم نمونه & نوع مطالعه & شركت كثندكّان & زمان انجام & نام نويسنده و سال انتشار & رديف \\
\hline iv & ينسيلوائيا & $m$ & ييمايش & بالاى ه\& سال & 1997 & كساب و همكاران (I998) [Tr] & 1 \\
\hline if & ألمان & DIE & مقطعى & بالاى VA سال & 199\% & ليثلن و همكاران (199V) ["T]] & r \\
\hline 10 & ايالت بايثيست كاروليناي & Impto & يبيمايش & ( & 1991 ட 199. & 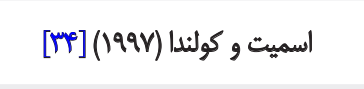 & r \\
\hline 19 & تايلند & pre. & بيمايش & بالاى •\& سال & 1990 & جيتايونكول و همكاران (1999) [به] & e \\
\hline r. & فيلاند & $i v \cdot V$ & مقطعى & (2) Vq-8. & 1994 51994 & [IV] (1999) تورو و همكاران & $\Delta$ \\
\hline ir & هند & eqd & ييمايش & بالاى هـ سال & $r+l_{1}$ & 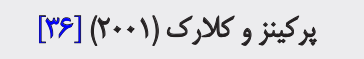 & 8 \\
\hline if & ميشيكان & slar & 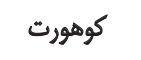 & بالاى V. سال & $199 \Delta, 1994$ & 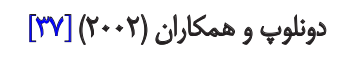 & $\gamma$ \\
\hline iv & ايالت منيتوبا كانادا & MAD & مطالعه طولى & 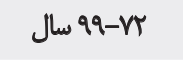 & $r+1 \sqcup|9 r|$ & 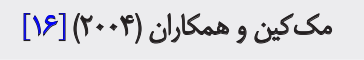 & $\wedge$ \\
\hline 10 & آوكسبورى آلمان & rA. & 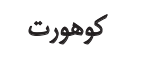 & - & 1994 ๒ 1999 & 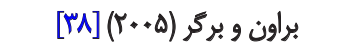 & 9 \\
\hline 18 & ايالت بريتيش كلمبيا & prim & مقطعى & بالاى هـ سال & 1999 E194 & 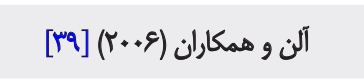 & 1. \\
\hline iv & البياثيا & VAY & مقطعى & بالاي ex whe & 1999 & 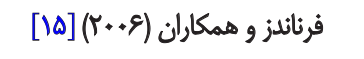 & 11 \\
\hline iv & تروز و فنلائد & จค19 & مقطعى & J Wh-8D & 1990 & 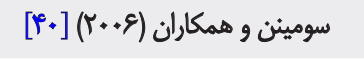 & ir \\
\hline 18 & ايالات متحله & men & مقطعى & ل & r.+r & [F) & ir \\
\hline 10 & 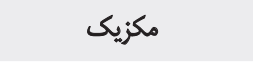 & ११. & مقطعى & بالاي .0 سال & r.+rEr..1 & بنجامينز (Y..V] & if \\
\hline r. & مكزيك & imp.. & كوهورت & بالاى .ه سال & $r+\cdot 1$ & 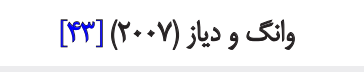 & 10 \\
\hline M & كانادا & rear & 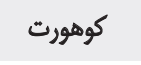 & بالاى .0 سال & $r+.151990$ & 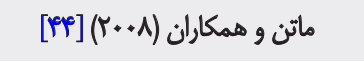 & is \\
\hline 18 & تورونتو در استان آنثاريو & Tra. & مقطعى & بالاي ه\& سال & r.॰QLE..P & 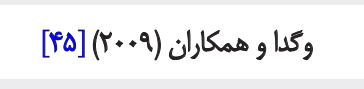 & iv \\
\hline 18 & مكزيك (شمال شرقى) & $r \cdot r \cdot$ & مقطعى & 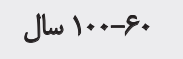 & $r++g t r+. r$ & دى كوردووا و همكاران (+1+r) [F"] & $M$ \\
\hline if & كائادا & ثامشتخص & مقطعيى & بالاى هـ سال & r..rLtr..r & 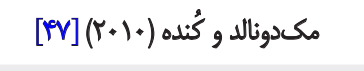 & 19 \\
\hline 18 & ايالات متحله & r...r & مقطعى & بالاى & r.AQLY..P & [بر & r. \\
\hline 10 & آلمان & 1.89 & كوهورت & 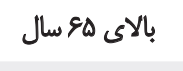 & Y.l. & 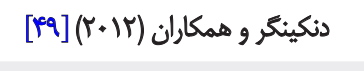 & m \\
\hline iv & s & $1+1+i$ & 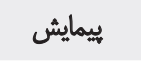 & بالاى به سال & $r+\Delta, r+\cdots 1$ & 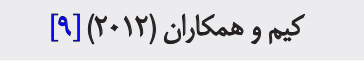 & $\pi$ \\
\hline if & ثنابوان & role & ييمايش & بالاي هاء سال & $r+\cdot r$ & 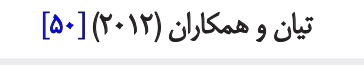 & r \\
\hline 18 & اورشليم، فلسطين اشغالى & $r+e q$ & 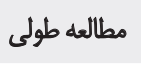 & J NA-VA & $r+1.5199$. & 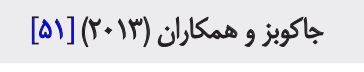 & re \\
\hline 19 & ايالات متحله & rafN & 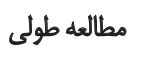 & بالاى •ه سال & $r++\Lambda t r++r$ & 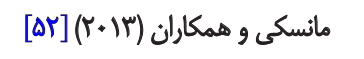 & ro \\
\hline r. & أيالت أميومالانكا در جنويى & $\Delta V 9 \Delta$ & مقطعى & بالاى .ه سال & r.l. & 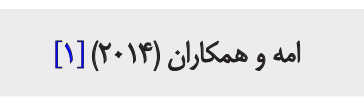 & re \\
\hline 18 & \& & $p e$ & مطالعه طولى & بالاي هo سال & $r+\infty$ & {$\left[\Delta T^{m}\right](r \cdot 1 f) \mathcal{S}$} & M \\
\hline r. & روسين، غناله هنذه، مكزيك. & re... & مطالعه طولى & بالاي +ه سال & $r+1 \cdot t r+r$ & 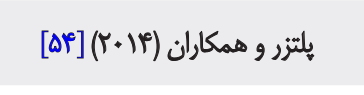 & ru \\
\hline M & 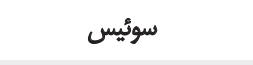 & TYqFq" & مقطعى & بالاى هo سال & $r+1 r$ & 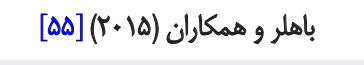 & rq \\
\hline iv & كائادا & DEgAY & مقطعى & بالاى • هال & $r \cdots \Delta$ & فيشر و همكاران (T) & $r$ \\
\hline
\end{tabular}




\begin{tabular}{|c|c|c|c|c|c|c|c|}
\hline اميّاز الرزيابى & محل انجام مطالعه & حجم نمونه & نوع مطالعه & شركت كنتدكان & زمان انجام & نام نويسنده و سال اتتشار & رديف \\
\hline iv & ايالات مثحله & rar. & ييمايش & بالاى •8 سال & $r \cdot I r, r \cdots A$ & 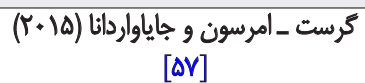 & m \\
\hline 19 & 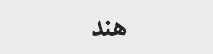 & MrATI & ييمايش & بالاى +ع سال & $r+r$ & 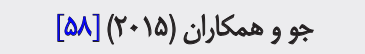 & rr \\
\hline 19 & rا كشور الرويائى & $\mid$ IXti & مقطعى مقى & بالاى +ه سال & $r \cdot+q u r+r$ & 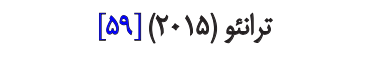 & M \\
\hline iv & اتيويى & TAF & همطعىي & بالاي •\& سال & $r \cdot 1 r$ & [r] & $m$ \\
\hline iv & جين & Aree & مطالعه طولى & بالاي fه سال & $r \cdot I_{r}$ & 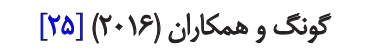 & ra \\
\hline 19 & ايالت بريثيش كلمبيا & $q Y \Delta q \cdot r$ & كوهورت & بالاي +ه سال & r..YEI99Y & 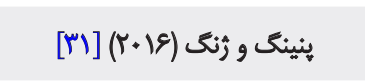 & re \\
\hline 18 & ألمان & rer. & يبيمايش & بالاى •ه سال & $r \cdot I V$ & 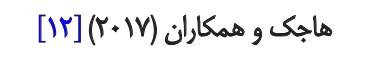 & $m$ \\
\hline iv & 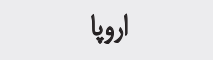 & AIYq & مقطعى & بالاي ه8 سال & Y.AL E..P & 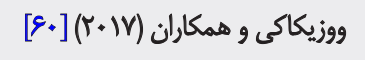 & ra \\
\hline iv & سمنانع ايران & mad & مقطعى & (9.-9 سال & $r+i r$ & 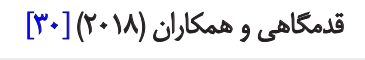 & $m q$ \\
\hline in & كره جنوبى & $r+1 q$ & ييمايش & 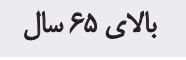 & $r+\Lambda, r \cdots q$ & 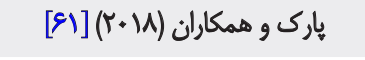 & f. \\
\hline 10 & كارائيب & 1.81 & مقطعى & بالاى •ع سال & $r \cdot M$ & 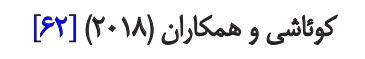 & il \\
\hline 19 & ايالت شائدونك جين & DQIf & مقطعى & بالاى •\& سال & $r \cdot I V$ & 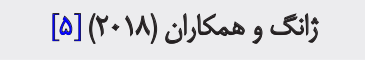 & RT \\
\hline 19 & 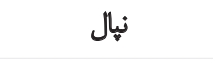 & $f+1$ & مقطعى & بالاى +ع بال & r.IV & 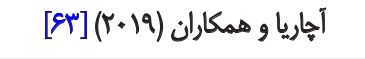 & m \\
\hline r. & ويتنام & ATr & هقطعى & بالاى •ع بال & $r+I r$ & 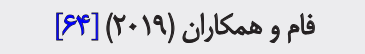 & $p$ \\
\hline
\end{tabular}

L

تحليل دادهاها

با توجه به هدف مرور سيستماثيك حاضر كه عوامل مرتبط با قراب

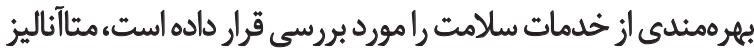

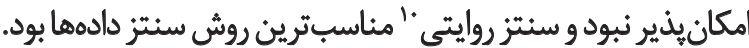

يافتهها

ويرُّكى هاي مطالعات

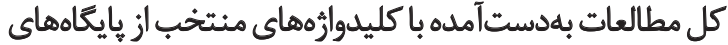

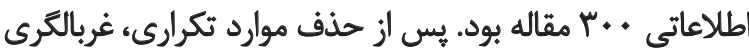

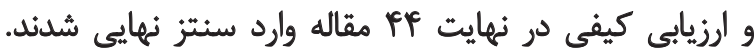

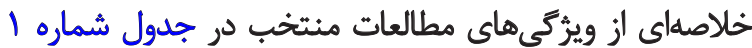

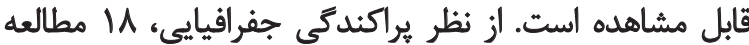

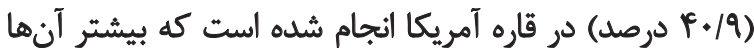

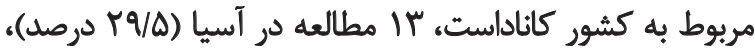

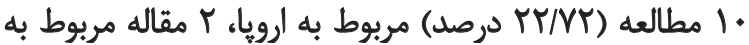

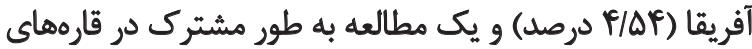

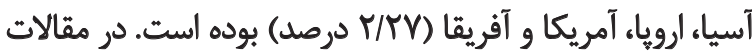

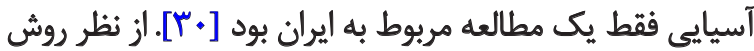

عنوان، جكيده، مقدمه (بيشينه و ضرورت مطالعه، اهداف)، روش

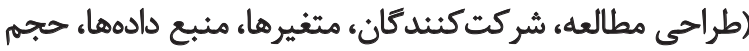

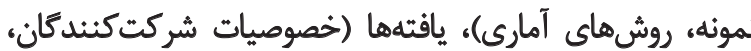

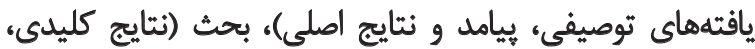

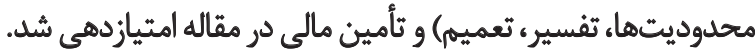

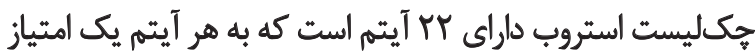

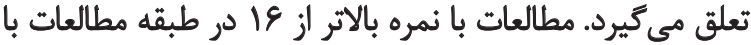

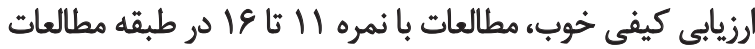

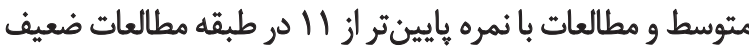

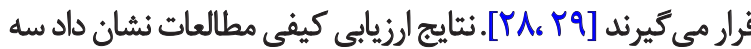

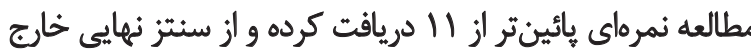

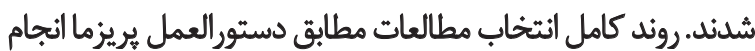
شد كه در تصوير شماره النمايش داده شده است.

\section{استخراج داده}

دادهها با استفاده از نرمافزار اكسل دستهبندى شدند. براي كليه

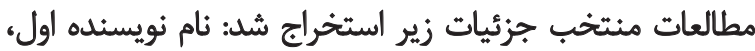

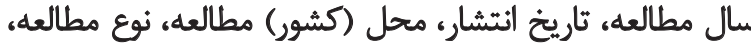

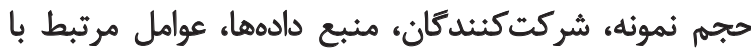
بهرهمندى از خدمات سلامت و امتياز ارزيابى كيفى داني. 


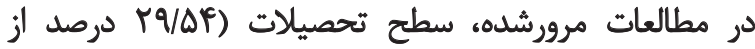

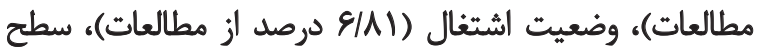

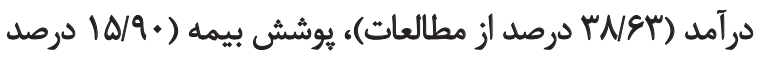

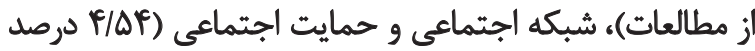

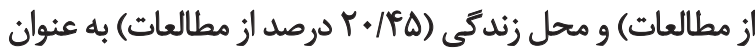

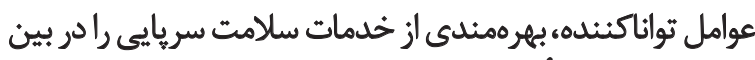

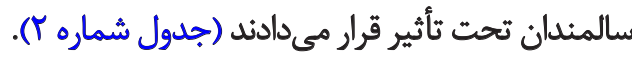

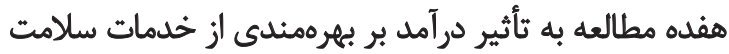

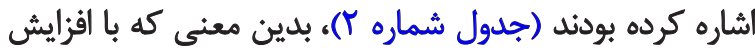

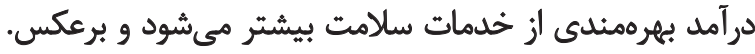

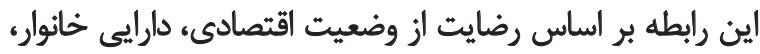

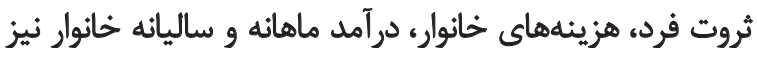

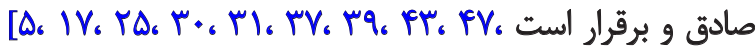

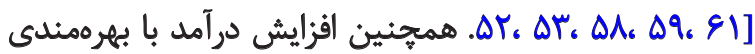

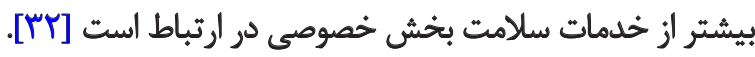

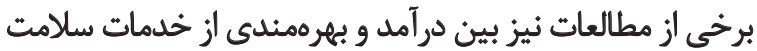

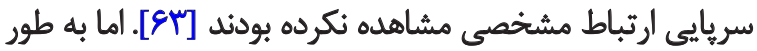

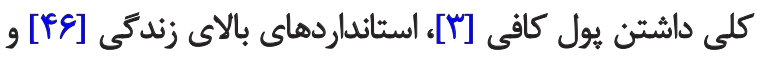

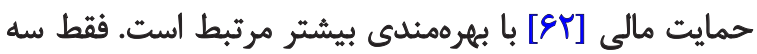

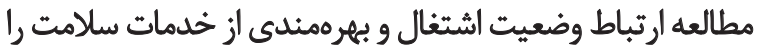

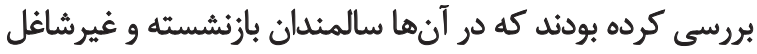

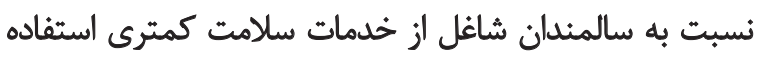

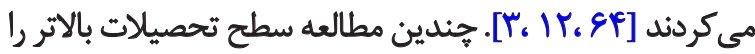

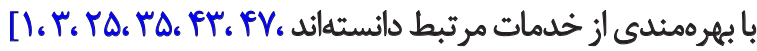

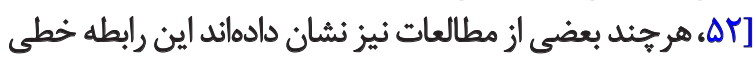

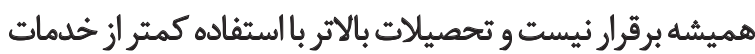

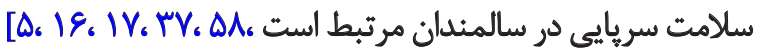

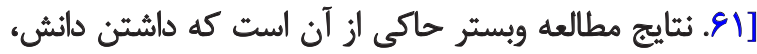

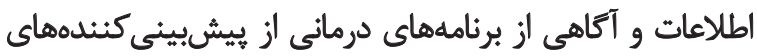

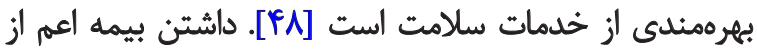

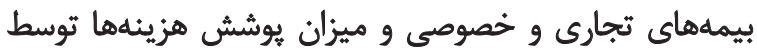

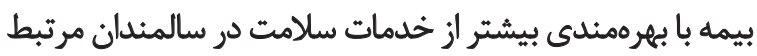

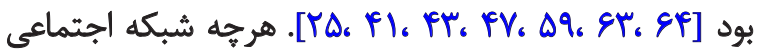

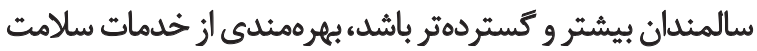

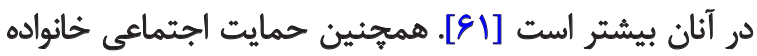

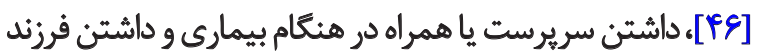

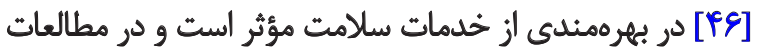

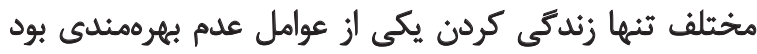

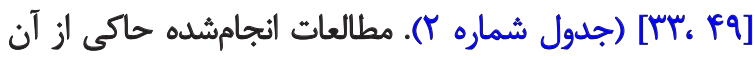

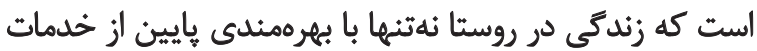

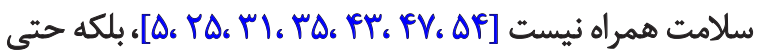

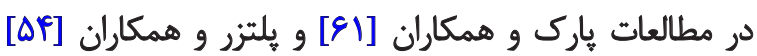

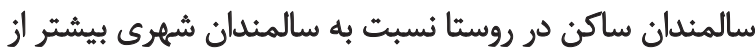

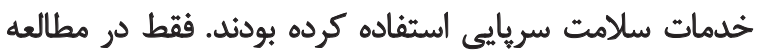

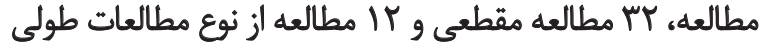

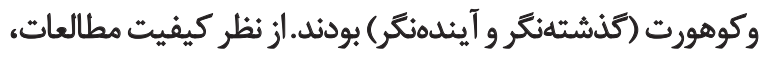

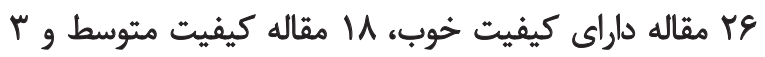

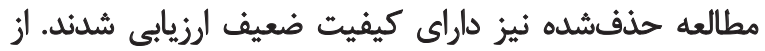

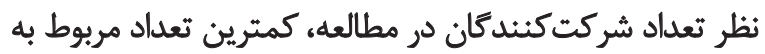

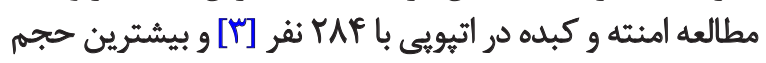

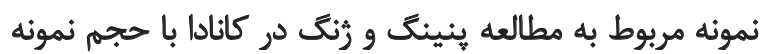

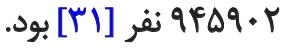

تعيين كثئدهاي بهرهمندى أز خُمات سلامت سريايى در سالمندان مدل رفتارى بهرهمندى از خدمات سلامت اندرسون به عنوان

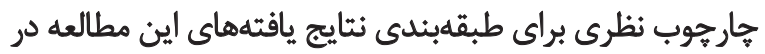

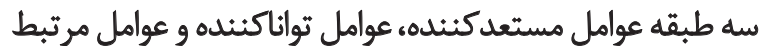

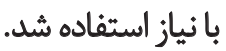

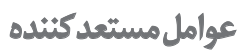

تقريباً در تمامى مطالعات مرورشده، عوامل مستعدكنيده از جراز

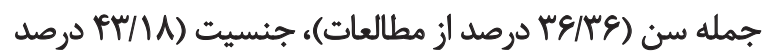

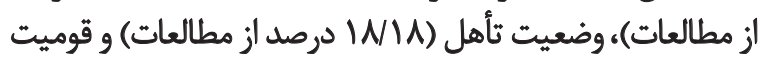

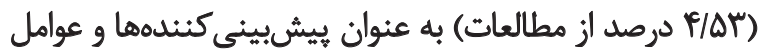

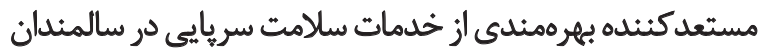

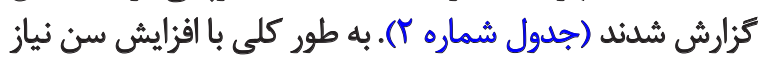

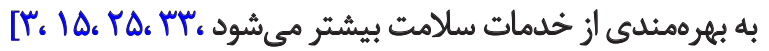

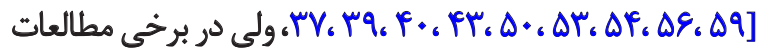

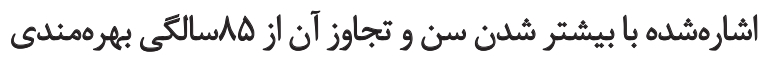

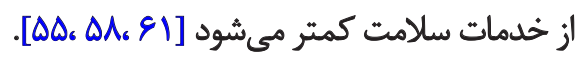

جنسيت با بهرهمندى از خدمات سلامت مرتبط بود، به طورى

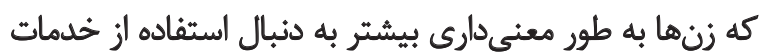

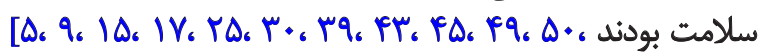

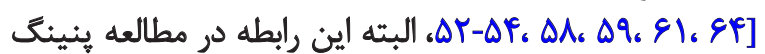

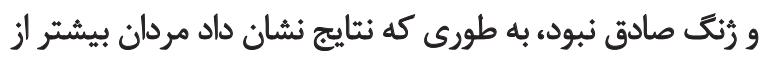

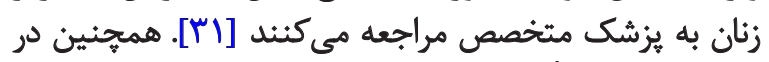

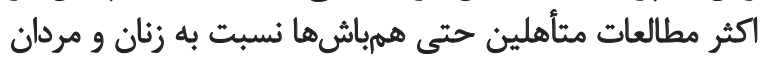

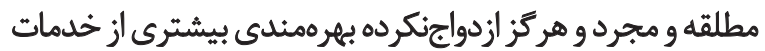

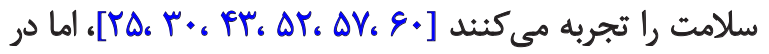

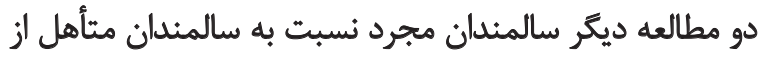

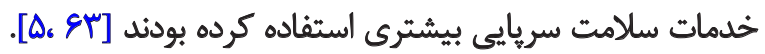

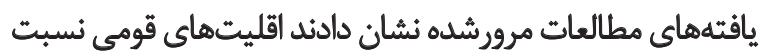

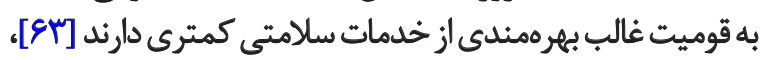

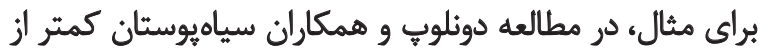

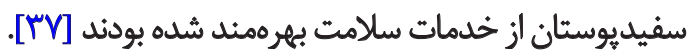

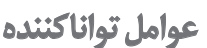


جدول Y. عوامل مؤثر بر بهرهمئدى از خدمات سلامت سريايى سالمندان در مطالعات مرورشده

\begin{tabular}{|c|c|}
\hline تعداد مطالعات & عوامل مؤثر بر بهرهمندى از خدمات سلامت سريايى سالمندان \\
\hline 19 & 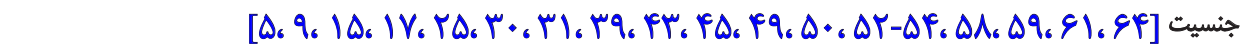 \\
\hline 18 & 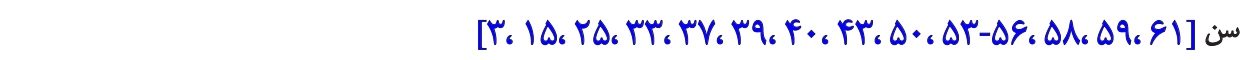 \\
\hline$\wedge$ & 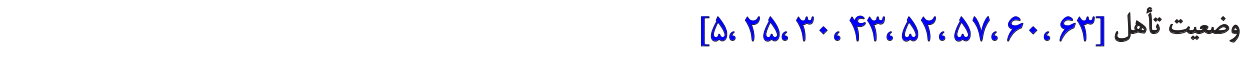 \\
\hline r & 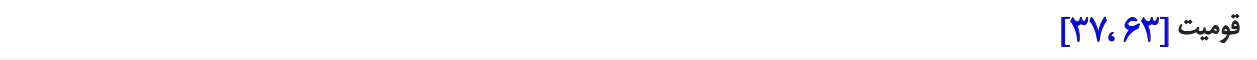 \\
\hline iv & 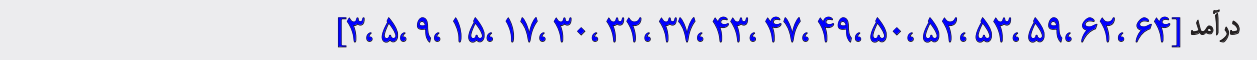 \\
\hline$\checkmark$ & {$\left[Y \Delta_{6} f f_{.} f r_{6} f V_{6} \Delta q_{6} g r_{6}, g f\right]$ بيمه } \\
\hline$\pi$ & 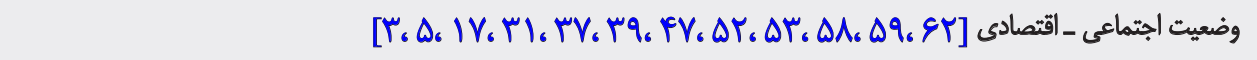 \\
\hline ir & {$\left[1, r_{6} \Delta_{6}\left|Y_{6}\right| V_{6} r \Delta_{6} r \Delta_{6} r Y_{6} f r_{6}\left|f V_{6} \Delta Y_{6} \Delta \Lambda_{6} \&\right|\right]$ سطح تحصيلات } \\
\hline r & 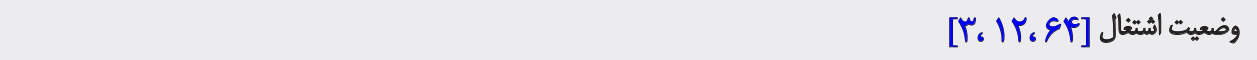 \\
\hline 1. & 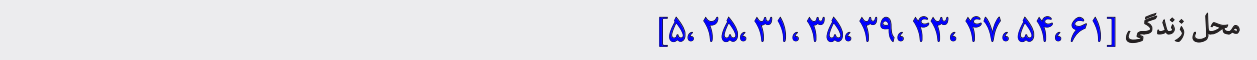 \\
\hline 1 & شبكه اجتماعى [ [ [ ] \\
\hline 1 & حمايت اجتماعى [fe \\
\hline r & 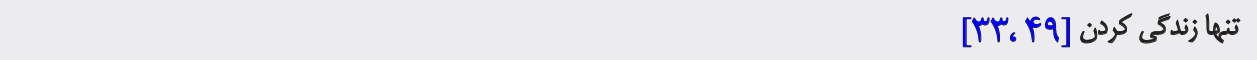 \\
\hline r & فاصله / عسافت تا مراكز ارائه خدمات سلامت [Y.F. [T] \\
\hline 1 & داشتن سريرست يا همراه در هنكام بيمارى [F9] \\
\hline 1 & 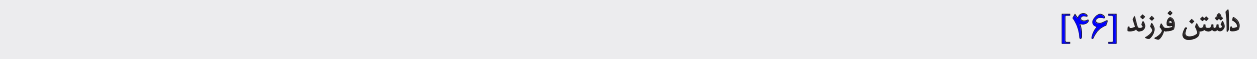 \\
\hline 1 & 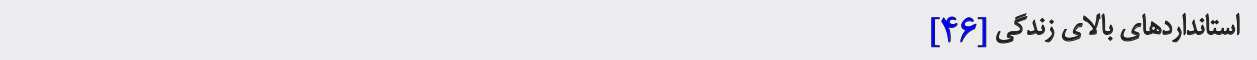 \\
\hline 1 & [ائش و اطلاعات سلامتى [FA] \\
\hline 1. & 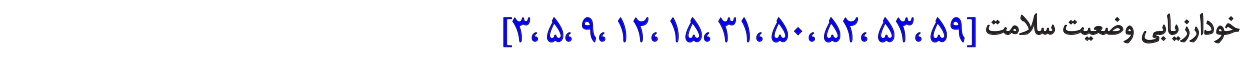 \\
\hline in & نو بيمارى (حاد يا مزمن) /طول مدت بيمارى [1، \\
\hline$\&$ & 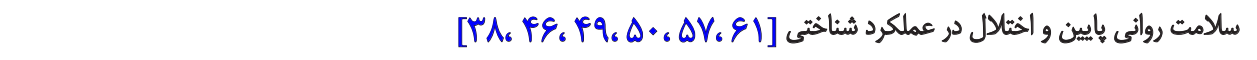 \\
\hline 1 & ناثوائى جسمى و محلوديت حركتي [YDD] \\
\hline r & 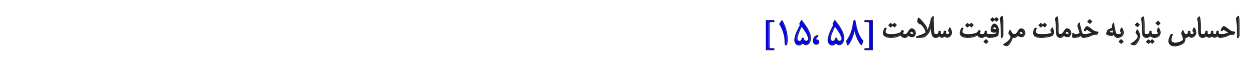 \\
\hline 1 & داشتن همزمان دو يا جند بيمارى [DQ ] \\
\hline$r$ & 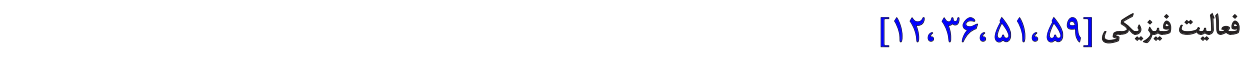 \\
\hline r & الحساس درد [Y\&، \\
\hline 1 & درى صحيح از وجود هشكل [ff] \\
\hline 1 & سبك زندكى (مصرف دخانيات، موادو الكل) [Yه ] \\
\hline
\end{tabular}

Uـ

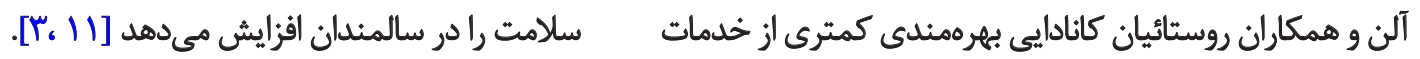

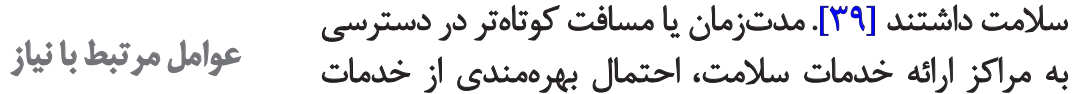


سلامت باشيم. يكى از اين راه حل ها، اصلاح نظام مراقبتهاى

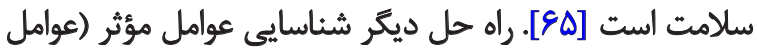

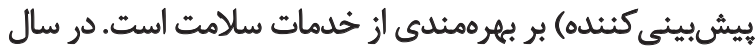

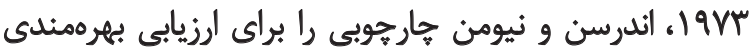

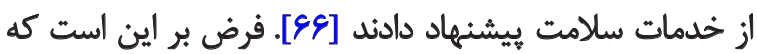

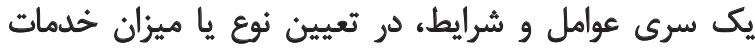

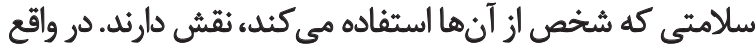

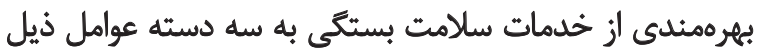

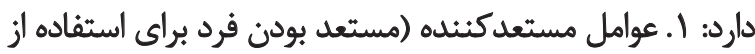

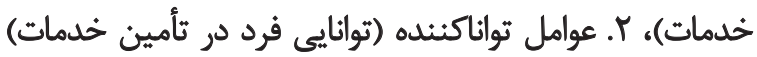

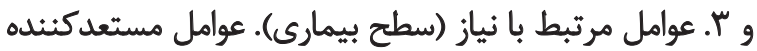

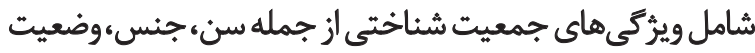

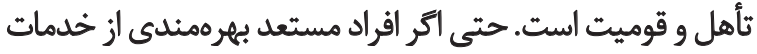

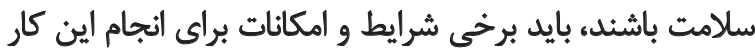

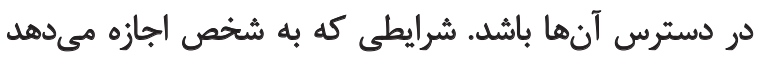

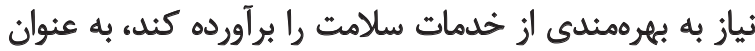

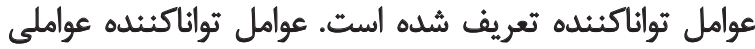

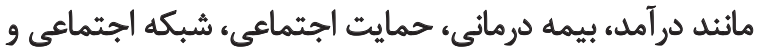

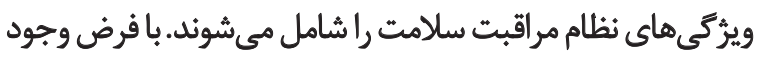

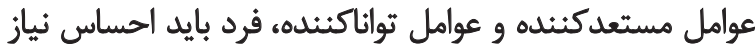

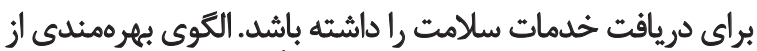

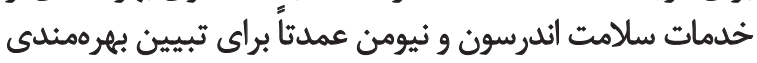

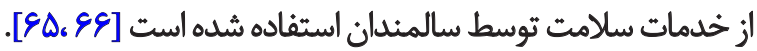

مرور مطالعات انجامشده به درك ما از تعيينكنيدهائ مائ

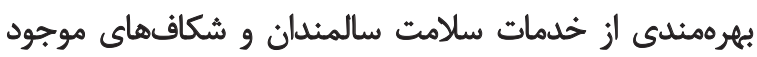

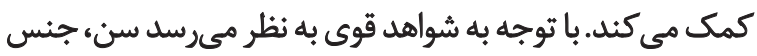

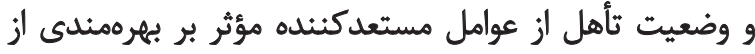

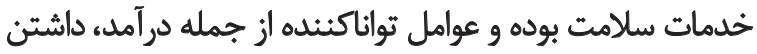

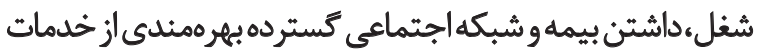

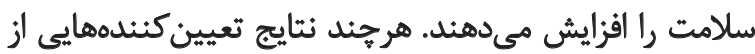

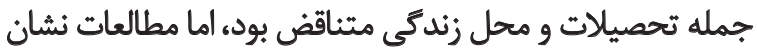

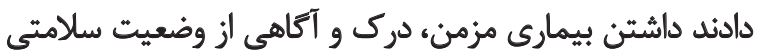

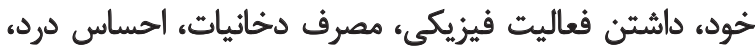

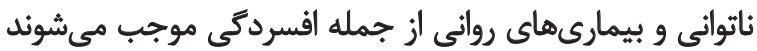

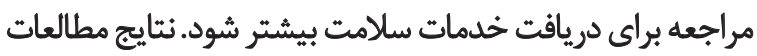

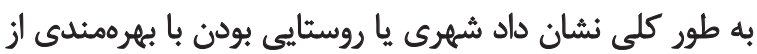

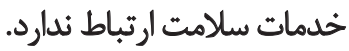

ماهيه طور كلى بر اساس يافتههاي اين مطالعه، داشتن درآمد

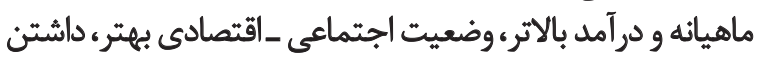

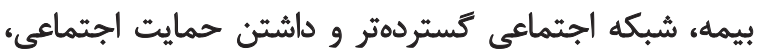

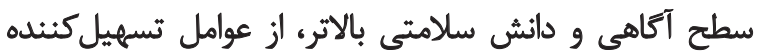

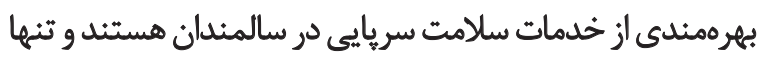

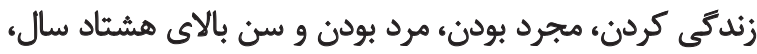

سنتز كلى يافتهها نشان داد عواملى مانند خودارزيابى از وضعيت

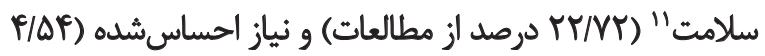

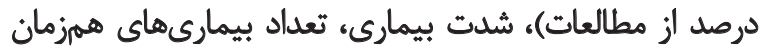

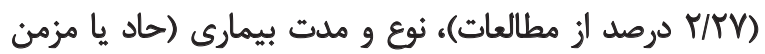

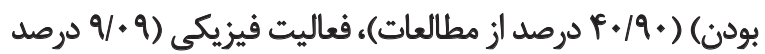

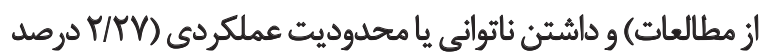

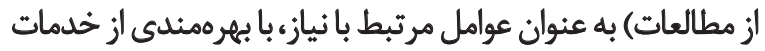

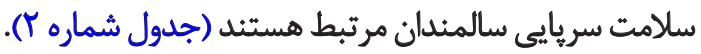

افراد داراى بيمارىهاى مزمن (حكلوكوم، ديابت، سرطان، فشار

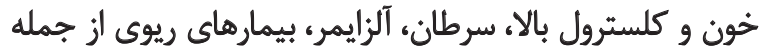

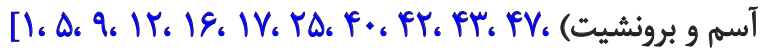

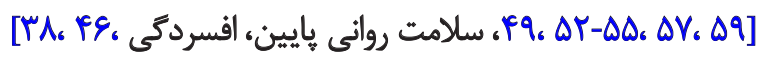

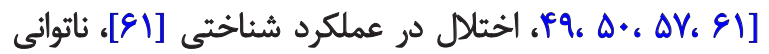

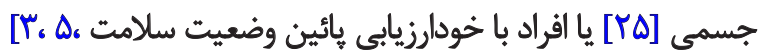

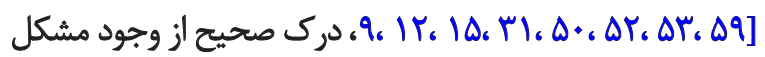

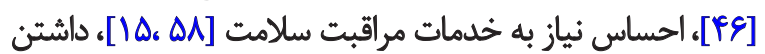

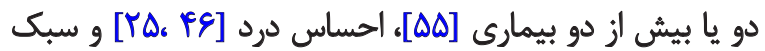

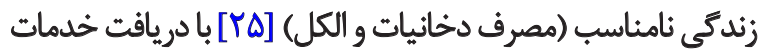

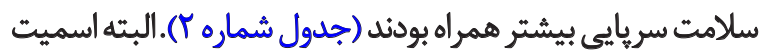

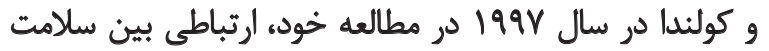

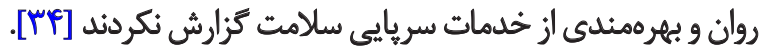

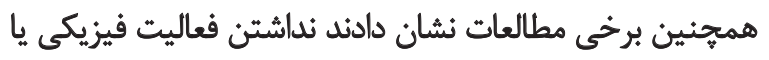

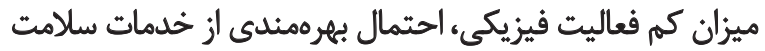

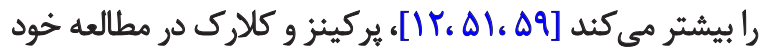

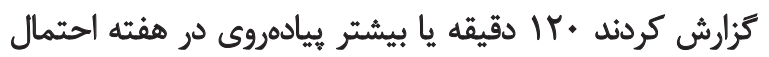

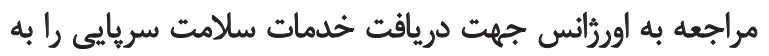

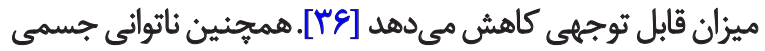

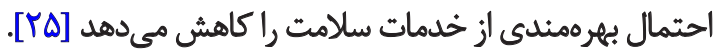

ثب

خلاصه يافتهاى اصلى

بجبود شرايط زندگى و افزايش طول عمر و اميد به زندگى إنى

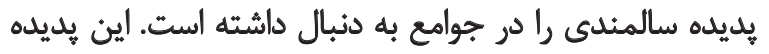

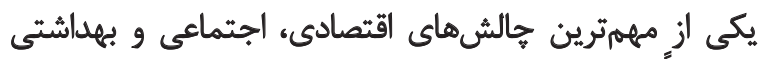

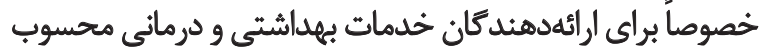

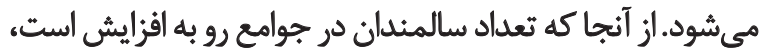

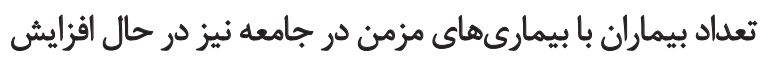

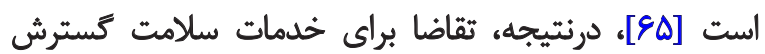

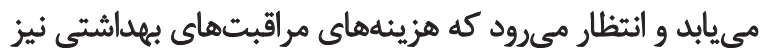

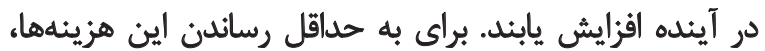

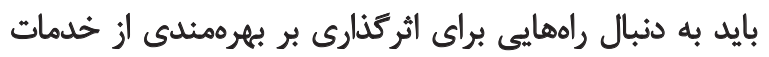

11. Self-assessed health status 
از سالمندان ارائه شود. مطابق با مطالعات ييشين بيمارى روانى إئى

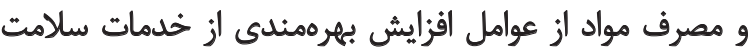

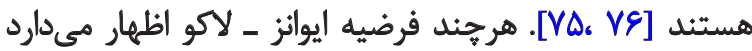

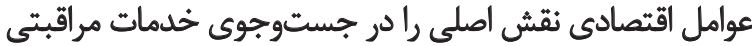

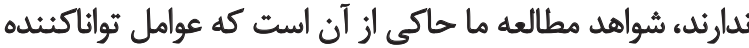

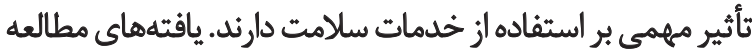

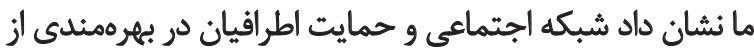

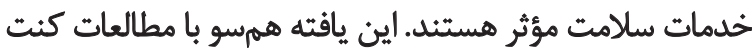

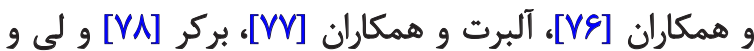

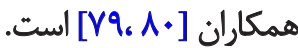

ممكن است تصميم در مورد مراجعه و بهرهمندى از خدمات

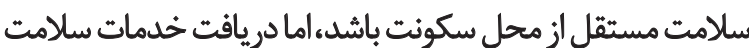

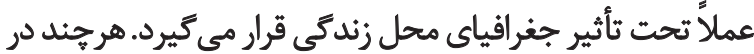

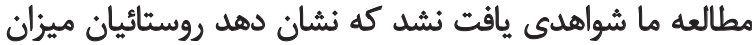

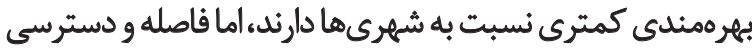

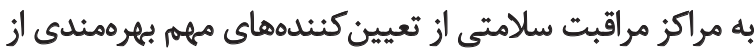

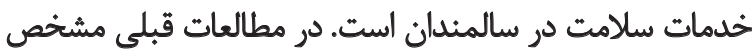

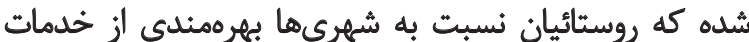

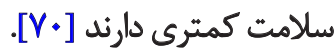

\section{نتيجلكَيرى نهايى}

اين مطالعه مرور سيستماتيك نشان داد مجموعادى از عوامل

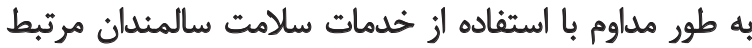

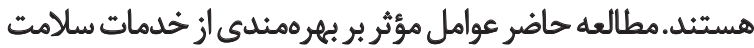
سالمندان را در سه دسته عوامل مستعدكنينده، تواناكنينده و نياز

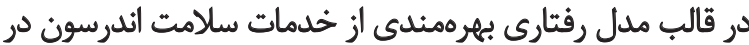

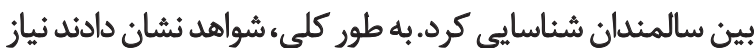

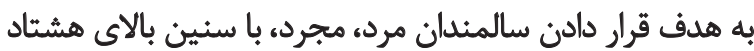

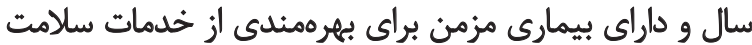

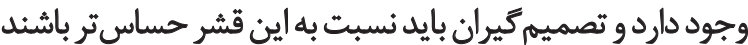

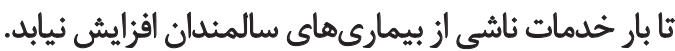

به نظر مىرسد مداخلات براي افزايش بهرهمندى از خحدمات سلامت

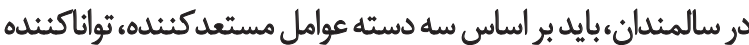

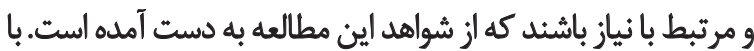

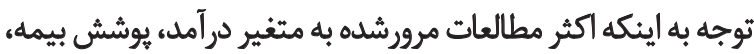

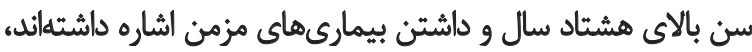

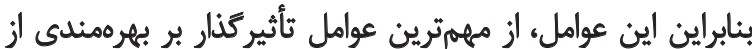

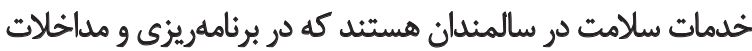

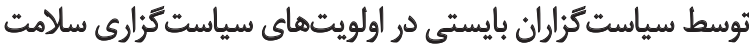
سالمندان مورد توجه قرار كيرند.

نقاط ضعف و قوت

از نقاط قوت اين مطالعه مىتوان به استفاده از استراتثرى
نداشتن فرزند، نداشتن همراه هنكام بيمارى، مدتز مان و ومسافت

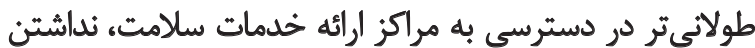

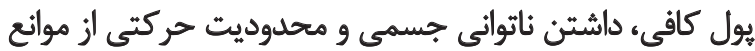

بهرهمندى ازٔ خدمات سريايى سلامت در سالمندان هستئد.

\section{مقايسه يافتهها با مطالعات قبلى}

همسو با مطالعات قبلى بررسى فده، در مطالعه مانيز مشخص

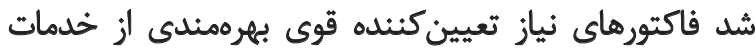

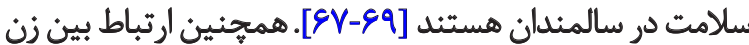

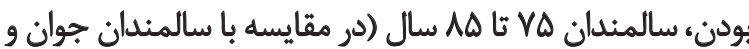

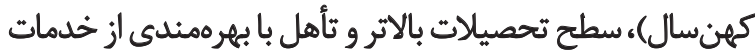

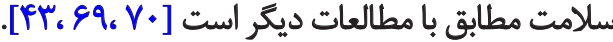

مطالعه ما هممو با مطالعه آندراده و همكاران [VI إن نشان داد

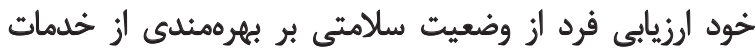

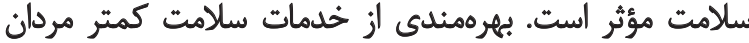

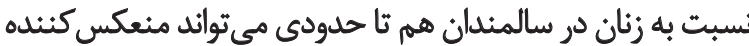

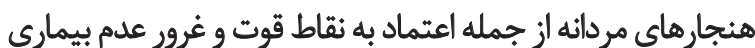

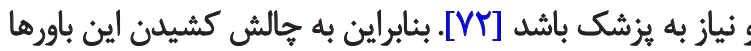

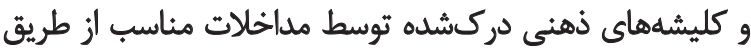

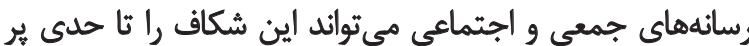

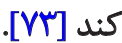

شواهد يافتشده از تأثير عوامل اقتصادى بر بهرهمندى از

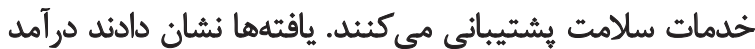

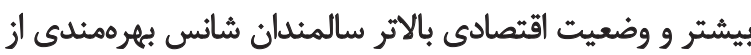

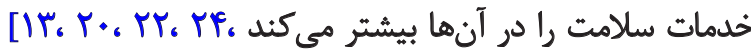

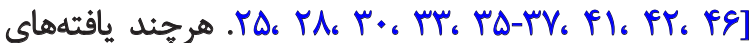

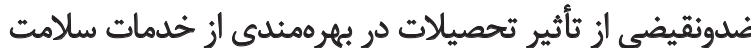

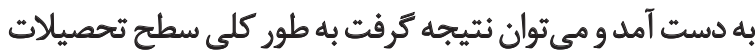

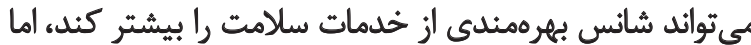

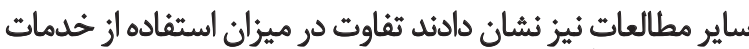

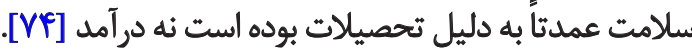
در مطالعه حاضر مشخص شد بيمارى روانى، افسردتى، مصرف

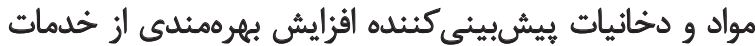

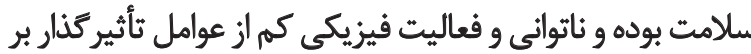

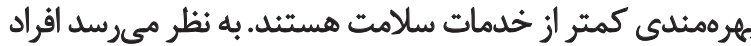

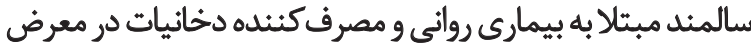

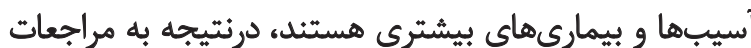

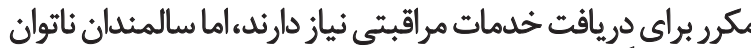

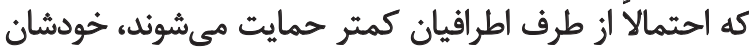

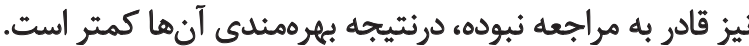

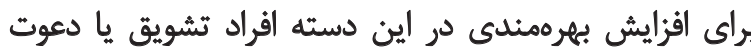

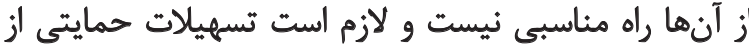

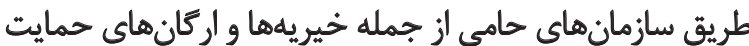


و تأثير كذار بر بهرهمندى از خدمات سلامت سالمندان هستند،

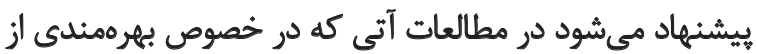

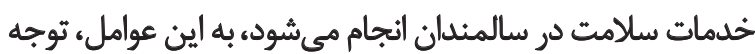

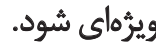

\section{ملاحظات اخلاقى \\ يبروى از اصول اخلاق يثوهش}

مطالعه حاضر با كد اخلاق IR.USWR.REC.1397.029 در ترد

دانشكاه علوم بهزيستى و توانبخشى تصويب شده است.

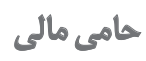

اين يرورُه توسط معاونت امور اجتماعى و فرهنكى شهردارى

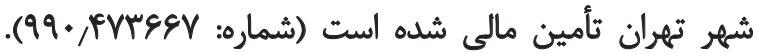

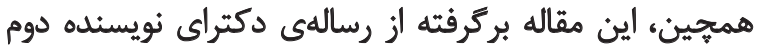

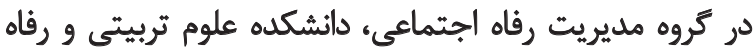
اجتماعى، دانشًاه علوم بجزيستى و توانبخشى، تهران است.

$$
\text { مشاركت ثويسندكًان }
$$

تمامى نويسندكان در طراحى مطالعه، متدولورئ و نكارش همه بخش هاى مطالعه حاضر مشاركت داشت مطانهاند.

$$
\text { تعارض مناقع }
$$

بنا به اظهار نويسندكان، در اين مقاله هيجَّونه تعارض منافعى

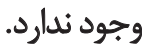

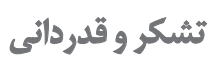

تمامى نويسندكان از راهنمايعها و حمايتهاى علمى استاد

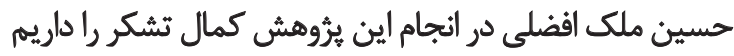

جستوجوى كسترده، بر اساس جستوجوى مطالعات قبلى و

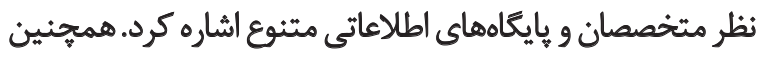

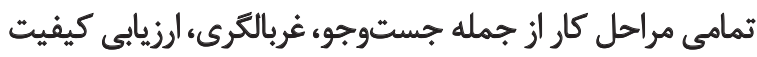

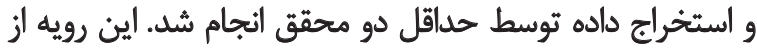

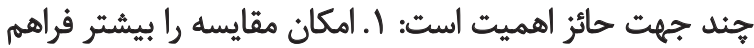

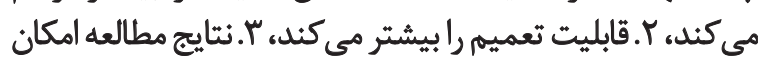

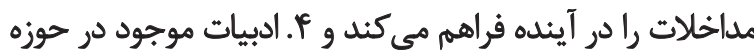

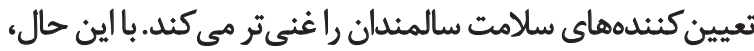

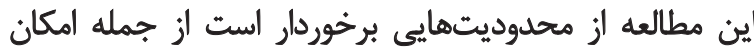

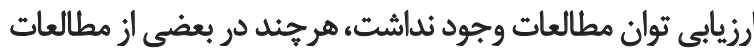

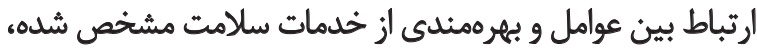

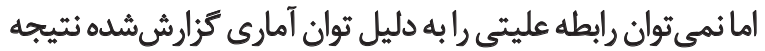

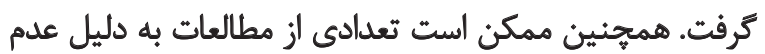

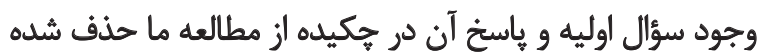

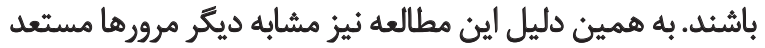

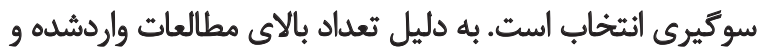

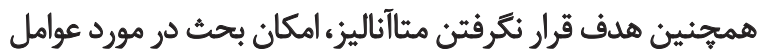
مخدوشكَر كنترل شده در مطالعات وجود ندارد.

با توجه به اينكه تعداد مطالعات فارسى كه به بر برسى عوامل بردي

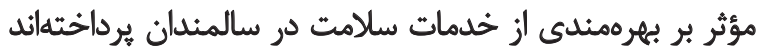

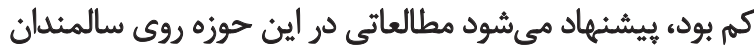

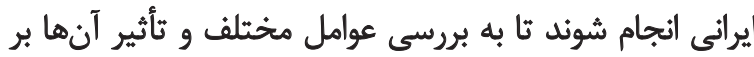

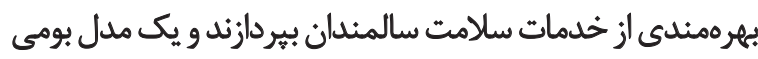

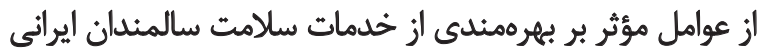

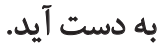

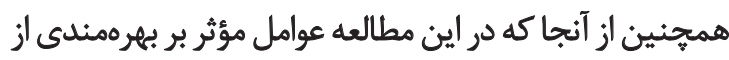

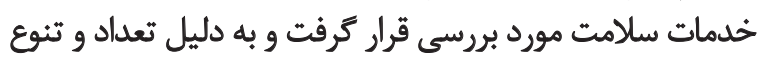

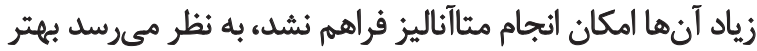

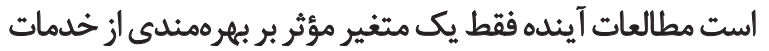

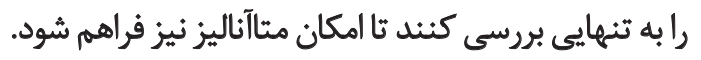

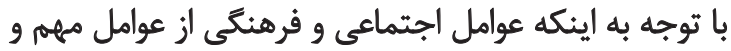

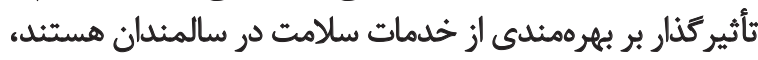

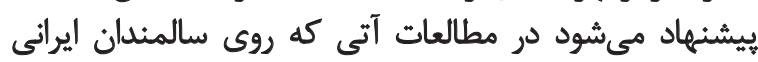

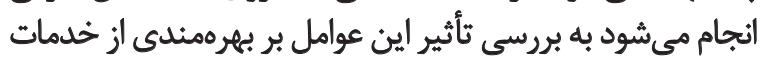
سلامت سالمندان برداخته شود.

بر اساس مدل اندرسون، باور و عقايد، عوامل فرهنكئ، عوامل

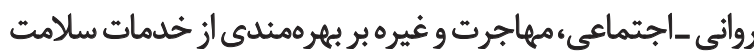

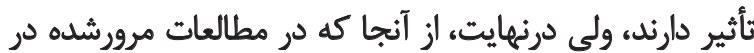

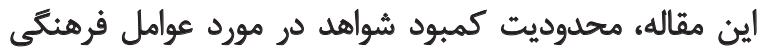

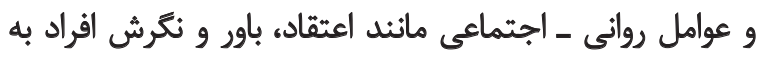

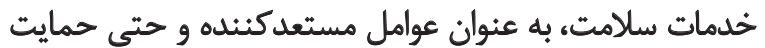

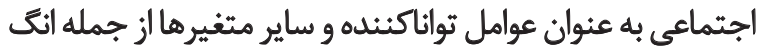

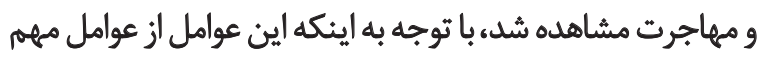




\section{References}

[1] Ameh S, Gómez-Olivé FX, Kahn K, Tollman SM, KlipsteinGrobusch K. Predictors of health care use by adults 50 years and over in a rural South African setting. Global Health Action. 2014; 7(1):24771. [DOI:10.3402/gha.v7.24771] [PMID] [PMCID]

[2] Fu X, Sun N, Xu F, Li J, Tang Q, \& He J, et al. Influencing factors of inequity in health services utilization among the elderly in China. International Journal for Equity in Health. 2018; 17(1):1-10. [DOI:10.1186/s12939-018-0861-6] [PMID] [PMCID]

[3] Amente T, Kebede B. Determinants of health service utilization among older adults in Bedele Town, Illubabor Zone, Ethiopia. Journal of Diabetes and Metabolism. 2016; 7(11):1000713. [DOI:10.4172/2155-6156.1000713]

[4] Yavari K, Basakha M, Sadeghi H, Naseri AR. [Economic aspects of ageing (Persian)]. Salmand: Iranian Journal of Ageing. 2015;10(1):92-105. http://salmandj.uswr.ac.ir/article-1-702-en.html

[5] Zhang J, Xu L, Li J, Sun L, Ding G, Qin W, et al. Loneliness and health service utilization among the rural elderly in Shandong, China: A cross-sectional study. International Journal of Environmental Research and Public Health. 2018; 15(7):1468 [DOI:10.3390/ijerph15071468] [PMID] [PMCID]

[6] Saboor M, Akbari Kamrani AA, Abolfathi Momtaz Y, Sahaf R. Prevalence and associated factors of potentially inappropriate medications among Iranian older adults. Medicinski Glasnik. 2019; 16(1):121-7. [DOI:10.17392/989-19] [PMID]

[7] Shrivastava SRBL, Shrivastava PS, Ramasamy J. Healthcare of elderly: Determinants, needs and services. International Journal of Preventive Medicine. 2013; 4(10):1224-5. [PMID] [PMCID]

[8] Puska P, Kalache A. Towards policy for health and ageing [Internet]. 2002 [Updated 2002]. Available from: http:/ / www.who. int/ageing/publications/alc_fs_ageing_policy.pdf

[9] Kim D, Shin H, Kim Cy. Equitable access to health care for the elderly in South Korea: Is income-related inequality in health care utilization more pronounced? Research on Aging. 2012; 34(4):47596. [DOI:10.1177/0164027511423538]

[10] Momtaz YA, Hamid TA, Ibrahim R. Unmet needs among disabled elderly Malaysians. Social Science \& Medicine. 2012; 75(5):859-63. [DOI:10.1016/j.socscimed.2012.03.047] [PMID]

[11] Yam HK, Mercer SW, Wong LY, Chan WK, Yeoh EK. Public and private healthcare services utilization by non-institutional elderly in Hong Kong: Is the inverse care law operating? Health Policy. 2009; 91(3):229-38. [DOI: 10.1016/j.healthpol.2008.12.012] [PMID]

[12] Hajek A, Bock JO, Konig HH. Which factors affect health care use among older Germans? Results of the German ageing survey. BMC Health Services Research. 2017; 17(1):30. [DOI:10.1186/ s12913-017-1982-0] [PMID] [PMCID]

[13] Babitsch B, Gohl D, von Lengerke T. Re-revisiting Andersen's behavioral model of health services use: A systematic review of studies from 1998-2011. GMS Psycho-Social-Medicine. 2012; 9:Doc11. [DOI:10.3205/psm000089] [PMID] [PMCID]

[14] Yang PQ, Hwang SH. Explaining immigrant health service utilization: A theoretical framework. SAGE Open. 2016; 6(2). [DOI:10.1177/2158244016648137]

[15] Fernández-Olano C, Hidalgo JD, Cerdá-Díaz R, RequenaGallego M, Sánchez-Castaño C, Urbistondo-Cascales L, et al.
Factors associated with health care utilization by the elderly in a public health care system. Health Policy. 2006; 75(2):131-9. [DOI:10.1016/j.healthpol.2005.02.005] [PMID]

[16] McKeen NA, Chipperfield JG, Campbell DW. A longitudinal analysis of discrete negative emotions and health-services use in elderly individuals. Journal of Aging and Health. 2004; 16(2):204 27. [DOI:10.1177/0898264303262648] [PMID]

[17] Noro AM, Häkkinen UT, Laitinen OJ. Determinants of health service use and expenditure among the elderly Finnish population. European Journal of Public Health. 1999; 9(3):174-80 [DOI:10.1093/eurpub/9.3.174]

[18] Nooraiee Motlagh S, Saber Mahani A, Barooni M, Asadi Lari M, Vaez Mahdavi MR, Hadian M. [Determining factors related to health services utilization (Persian)]. Razi Journal of Medical Sciences. 2015; 21(127):61-72. http://rjms.iums.ac.ir/article1-3475-en.html

[19] Chiavegatto Filho AD, Wang YP, Malik AM, Takaoka J, Viana MC, Andrade LH. Determinants of the use of health care services: Multilevel analysis in the Metropolitan Region of Sao Paulo Revista de Saúde Pública. 2015; 49:15. [DOI:10.1590/S00348910.2015049005246] [PMID] [PMCID]

[20] Borhaninejad VR, Naghibzadeh Tahami A, Nabavi H, Rashedi V, Yazdi-Feyzabadi V. [The utilization of health services and its influences among elderly people in Kerman-2014 (Persian)] Journal of North Khorasan University of Medical Sciences. 2015 7(2):229-40. [DOI:10.29252/jnkums.7.2.229]

[21] SoleimanvandiAzar N, Mohaqeqi Kamal SH, Sajjadi H, Ghaedamini Harouni GR, Karimi SE, Djalalinia S, et al. Determinants of outpatient health service utilization according to andersens behavioral model: A systematic scoping review. Iranian Journal of Medical Sciences. 2020; 45(6):405-24. [DOI: 10.30476/ ijms.2020.85028.1481] [PMID] [PMCID]

[22] Ghaedamini Harouni GR, Sajjadi H, Rafiey H, Mirabzadeh A Vaez-Mahdavi MR, Mohaqeqi Kamal SH. Current status of health index in Tehran: A multidimensional approach. Medical Journal of the Islamic Republic of Iran. 2017; 31:29. [DOI:10.18869/ mjiri.31.29] [PMID] [PMCID]

[23] Filipski MJ, Zhang Y, Chen KZ. Making health insurance propoor: Evidence from a household panel in rural China. BMC Health Services Research. 2015; 15:210. [DOI:10.1186/s12913015-0871-7] [PMID] [PMCID]

[24] Gotsadze G, Murphy A, Shengelia N, Zoidze A. Healthcare utilization and expenditures for chronic and acute conditions in Georgia: Does benefit package design matter? BMC Health Services Research. 2015; 15:88. [DOI:10.1186/s12913-015-0755-x] [PMID] [PMCID]

[25] Gong CH, Kendig H, He X. Factors predicting health services use among older people in China: An analysis of the China health and retirement longitudinal study 2013. BMC Health Services Research. 2016; 16:63. [DOI:10.1186/s12913-016-1307-8] [PMID] [PMCID]

[26] Abera Abaerei A, Ncayiyana J, Levin J. Health-care utilization and associated factors in Gauteng province, South Africa. Global Health Action. 2017; 10(1):1305765. [DOI:10.1080/16549716.2017 .1305765] [PMID] [PMCID]

[27] Mohammadbeigi A, Hassanzadeh J, Eshrati B, Rezaianzadeh A. Socioeconomic inequity in health care utilization, Iran. Journal of Epidemiology and Global Health. 2013; 3(3):139-46. [DOI:10.1016/j.jegh.2013.03.006] [PMID] [PMCID] 
[28] Vandenbroucke JP, Von Elm E, Altman DG, Gøtzsche PC, Mulrow $C D$, Pocock SJ, et al. Strengthening the Reporting of Observational Studies in Epidemiology (STROBE): Explanation and elaboration. Annals of Internal Medicine. 2007; 147(8):W-163-W-94. [DOI:10.7326/0003-4819-147-8-200710160-00010] [PMID]

[29] von Elm E, Altman DG, Egger M, Pocock SJ, Gøtzsche PC, Vandenbroucke JP. The Strengthening the Reporting of Observational Studies in Epidemiology (STROBE) statement: guidelines for reporting observational studies. Annals of Internal Medicine. 2007; 147(8):5737. [DOI:10.7326/0003-4819-147-8-200710160-00010] [PMID]

[30] Ghadamgahi HB, Norouzi K, Mohammadi F, Norouzi M, Jandaqhi J. [Status and determinants of health services utilization among elderly rural habitants in the Iraninan population (Persian)]. Koomesh. 2018; 20(4):779-85. http://koomeshjournal. semums.ac.ir/article-1-4184-en.html

[31] Penning MJ, Zheng C. Income inequities in health care utilization among adults aged 50 and older. Canadian Journal on Aging. 2016; 35(1):55-69. [DOI:10.1017/S0714980815000562] [PMID]

[32] Kassab C, Luloff AE, Kelsey TW, Smith SM. The influence of insurance status and income on health care use among the nonmetropolitan elderly. Journal of Rural Health. 1996; 12(2):89-99. [DOI:10.1111/j.1748-0361.1996.tb00780.x] [PMID]

[33] Linden M, Horgas AL, Gilberg R, Steinhagen-Thiessen E. Predicting health care utilization in the very old: The role of physical health, mental health, attitudinal and social factors. Journal of Aging and Health. 1997; 9(1):3-27. [DOI:10.1177/089826439700900 101] [PMID]

[34] Smith SL, Colenda CC. Mental health and health care utilization in geriatric primary care patients. International Journal of Psychiatry in Medicine. 1997; 27(1):23-32. [DOI:10.2190/WU2ARTV9-G3V4-Q5V4] [PMID]

[35] Jitapunkul S, Na Songkhla M, Chayovan N, Chirawatkul A, Choprapawon C, Kachondham Y, et al. A national survey of health-service use in Thai elders. Age and Ageing. 1999; 28(1):6771. [DOI:10.1093/ageing/28.1.67] [PMID]

[36] Perkins AJ, Clark DO. Assessing the association of walking with health services use and costs among socioeconomically disadvantaged older adults. Preventive Medicine. 2001; 32(6):492501. [DOI:10.1006/pmed.2001.0832] [PMID]

[37] Dunlop DD, Manheim LM, Song J, Chang RW. Gender and ethnic/racial disparities in health care utilization among older adults. Journals of Gerontology - Series B Psychological Sciences and Social Sciences. 2002; 57(4):S221-33. [DOI:10.1093/ geronb/57.4.S221] [PMID]

[38] Braune BT, Berger K. The influence of depressive mood on activities of daily living and health care utilization in the elderly - The MEMO study on the KORA platform Augsburg. Gesundheitswesen. 2005; 67 Suppl 1:S176-9. [DOI:10.1055/s-2005-858236] [PMID]

[39] Allan D, Cloutier-Fisher D. Health service utilization among older adults in British Columbia: Making sense of geography. Canadian Journal on Aging. 2006; 25(2):219-32. [DOI:10.1353/ cja.2006.0032] [PMID]

[40] Suominen-Taipale AL, Martelin T, Koskinen S, Holmen J, Johnsen R. Gender differences in health care use among the elderly population in areas of Norway and Finland. A crosssectional analysis based on the HUNT study and the FINRISK Senior Survey. BMC Health Services Research. 2006; 6:110. [DOI:10.1186/1472-6963-6-110] [PMID] [PMCID]
[41] Xu X, Patel DA, Vahratian A, Ransom SB. Insurance coverage and health care use among near-elderly women. Women's Health Issues. 2006;16(3):139-48. [DOI:10.1016/j.whi.2006.02.005] [PMID]

[42] Benjamins MR. Predictors of preventive health care use among middle-aged and older adults in Mexico: The role of religion. Journal of Cross-Cultural Gerontology. 2007; 22(2):221-34. [DOI:10.1007/s10823-007-9036-4] [PMID]

[43] Wong R, Díaz JJ. Health care utilization among older Mexicans: Health and socioeconomic inequalities. Salud Pública de México. 2007;49 Suppl 4:S505-14. [DOI:10.1590/S003636342007001000010]

[44] Maaten S, Kephart G, Kirkland S, Andreou P. Chronic disease risk factors associated with health service use in the elderly. BMC Health Services Research. 2008; 8:237. [DOI:10.1186/1472-69638-237] [PMID] [PMCID]

[45] Vegda K, Nie JX, Wang L, Tracy CS, Moineddin R, Upshur REG. Trends in health services utilization, medication use, and health conditions among older adults: A 2-year retrospective chart review in a primary care practice. BMC Health Services Research. 2009; 9:217. [DOI:10.1186/1472-6963-9-217] [PMID] [PMCID]

[46] De Córdova MIP, Mier N, Curi EJM, Gómez TG, Quirarte NHG, Barrios FF. Personal and social determinants of health services utilization by Mexican older people. International Journal of Older People Nursing. 2010; 5(3):193-201. [DOI:10.1111/j.17483743.2009.00193.x] [PMID]

[47] McDonald JT, Conde H. Does geography matter? The health service use and unmet health care needs of older Canadians. Canadian Journal on Aging. 2010; 29(1):23-37. [DOI:10.1017/ S0714980809990389] [PMID]

[48] Webster NJ. Medicare knowledge and health service utilization among older adults. In: Jacobs Kronenfeld J, editor. Access to Care and Factors that Impact Access, Patients as Partners in Care and Changing Roles of Health Providers. Bingley: Emerald Group Publishing Limited; 2011. p. 273-297. [DOI:10.1108/ S0275-4959(2011)0000029014]

[49] Denkinger MD, Lukas A, Herbolsheimer F, Peter R, Nikolaus T. Physical activity and other health-related factors predict health care utilisation in older adults. The ActiFE Ulm study. Zeitschrift für Gerontologie und Geriatrie. 2012; 45(4):290-7. [DOI:10.1007/ s00391-012-0335-1] [PMID]

[50] Tian WH, Liu TC, Chen CS, Liu LF, Tien JJ. The relationship between depressive symptoms and health service utilization for elderly people in Taiwan. Health Policy. 2012; 108(2-3):256-67. [DOI:10.1016/j.healthpol.2012.10.005] [PMID]

[51] Jacobs JM, Rottenberg Y, Cohen A, Stessman J. Physical activity and health service utilization among older people. Journal of the American Medical Directors Association. 2013; 14(2):125-9. [DOI:10.1016/j.jamda.2012.10.023] [PMID]

[52] Manski RJ, Moeller JF, Chen H, Schimmel J, St. Clair PA, Pepper JV. Patterns of older Americans' health care utilization over time. American Journal of Public Health. 2013; 103(7):1314-24. [DOI:10.2105/AJPH.2012.301124] [PMID] [PMCID]

[53] Park JM. Health status and health services utilization in elderly Koreans. International Journal for Equity in Health. 2014; 13:73. [DOI:10.1186/s12939-014-0073-7] [PMID] [PMCID]

[54] Peltzer K, Williams JS, Kowal P, Negin J, Snodgrass JJ, Yawson $\mathrm{A}$, et al. Universal health coverage in emerging economies: Find- 
ings on health care utilization by older adults in China, Ghana, India, Mexico, the Russian Federation, and South Africa. Global Health Action. 2014; 7(1):25314. [DOI:10.3402/gha.v7.25314] [PMID] [PMCID]

[55] Bähler C, Huber CA, Brüngger B, Reich O. Multimorbidity, health care utilization and costs in an elderly community-dwelling population: A claims data based observational study. BMC Health Services Research. 2015; 15:23. [DOI:10.1186/s12913-0150698-2] [PMID] [PMCID]

[56] Fisher KL, Harrison EL, Reeder BA, Sari N, Chad KE. Is selfreported physical activity participation associated with lower health services utilization among older adults? Cross-sectional evidence from the Canadian community health survey. Journal of Aging Research. 2015; 2015:425354. [DOI:10.1155/2015/425354] [PMID] [PMCID]

[57] Gerst-Emerson K, Jayawardhana J. Loneliness as a public health issue: The impact of loneliness on health care utilization among older adults. American Journal of Public Health. 2015; 105(5):10139. [DOI:10.2105/AJPH.2014.302427] [PMID] [PMCID]

[58] Joe W, Rudra S, Subramanian SV. Horizontal inequity in elderly health care utilization: Evidence from India. Journal of Korean Medical Science. 2015; 30(Suppl 2):S155-66. [DOI:10.3346/ jkms.2015.30.S2.S155] [PMID] [PMCID]

[59] Terraneo M. Inequities in health care utilization by people aged 50+: Evidence from 12 European countries. Social Science \& Medicine. 2015; 126:154-63. [DOI:10.1016/j.socscimed.2014.12.028] [PMID]

[60] Vozikaki M, Linardakis M, Philalithis A. Preventive health services utilization in relation to social isolation in older adults. Journal of Public Health. 2017; 25(5):545-56. [DOI:10.1007/s10389017-0815-2]

[61] Park S, Kang JY, Chadiha LA. Social network types, health, and health-care use among South Korean older adults. Research on Aging. 2018; 40(2):131-54. [DOI:10.1177/0164027516682814] [PMID]

[62] Quashie NT. Gender differences in health care utilization among older adults in Barbados. In: Kronenfeld JJ, editor. Gender, Women's Health Care Concerns and Other Social Factors in Health and Health Care. Bingley: Emerald Publishing Limited; 2018. p. 211-233. [DOI:10.1108/S0275-495920180000036012]

[63] Acharya S, Ghimire S, Jeffers EM, Shrestha N. Health care utilization and health care expenditure of Nepali older adults. Frontiers in Public Health. 2019; 7:24. [DOI:10.3389/ fpubh.2019.00024] [PMID] [PMCID]

[64] Pham T, Nguyen NTT, ChieuTo SB, Le Pham T, Nguyen TX, Nguyen HTT, et al. Sex differences in quality of life and health services utilization among elderly people in rural Vietnam. International Journal of Environmental Research and Public Health. 2019; 16(1):69. [DOI:10.3390/ijerph16010069] [PMID] [PMCID]

[65] de Boer AG, Wijker W, de Haes HC. Predictors of health care utilization in the chronically ill: A review of the literature. Health Policy. 1997; 42(2):101-15. [DOI:10.1016/S0168-8510(97)00062-6]

[66] Andersen R, Newman JF. Societal and individual determinants of medical care utilization in the United States. The Milbank Memorial Fund Quarterly Health and Society. 1973; 51(1):95-124. [DOI:10.2307/3349613] [PMID]

[67] Leaf PJ, Bruce ML, Tischler GL, Freeman Jr DH, Weissman MM, Myers JK. Factors affecting the utilization of specialty and general medical mental health services. Medical Care. 1988; 26(1):9-26. [DOI:10.1097/00005650-198801000-00002] [PMID]

[68] Graham A, Hasking P, Brooker J, Clarke D, Meadows G. Mental health service use among those with depression: An exploration using Andersen's behavioral model of health service use Journal of Affective Disorders. 2017; 208:170-6. [DOI:10.1016/j. jad.2016.08.074] [PMID]

[69] Roberts T, Esponda GM, Krupchanka D, Shidhaye R, Patel V, Rathod S. Factors associated with health service utilisation for common mental disorders: a systematic review. BMC Psychiatry. 2018 18(1):262. [DOI:10.1186/s12888-018-1837-1] [PMID] [PMCID]

[70] Twomey CD, Baldwin DS, Hopfe M, Cieza A. A systematic review of the predictors of health service utilisation by adults with mental disorders in the UK. BMJ Open. 2015; 5(7):e007575. [DOI:10.1136/bmjopen-2015-007575] [PMID] [PMCID]

[71] Andrade LH, Alonso J, Mneimneh Z, Wells J, Al-Hamzawi A Borges $G$, et al. Barriers to mental health treatment: Results from the WHO World Mental Health surveys. Psychological Medicine. 2014; 44(6):1303-17. [DOI:10.1017/S0033291713001943] [PMID] [PMCID]

[72] Berger JL, Addis ME, Green JD, Mackowiak C, Goldberg V. Men's reactions to mental health labels, forms of help-seeking, and sources of help-seeking advice. Psychology of Men \& Masculinity. 2013; 14(4):433-43. [DOI:10.1037/a0030175]

[73] Martínez-Hernáez A, DiGiacomo SM, Carceller-Maicas N Correa-Urquiza M, Martorell-Poveda MA. Non-professionalhelp-seeking among young people with depression: A qualitative study. BMC Psychiatry. 2014; 14:124. [DOI:10.1186/1471 244X-14-124] [PMID] [PMCID]

[74] Evans-Lacko S, Aguilar-Gaxiola S, Al-Hamzawi A, Alonso J, Benjet C, Bruffaerts R, et al. Socio-economic variations in the mental health treatment gap for people with anxiety, mood, and substance use disorders: Results from the WHO World Mental Health (WMH) surveys. Psychological Medicine. 2018; 48(9):1560 71. [DOI:10.1017/S0033291717003336] [PMID] [PMCID]

[75] Fleury MJ, Grenier G, Bamvita JM, Caron J. Determinants and patterns of service utilization and recourse to professionals for mental health reasons. BMC Health Services Research. 2014 14(1):161. [DOI:10.1186/1472-6963-14-161] [PMID] [PMCID]

[76] Kent S, Fogarty M, Yellowlees P. A review of studies of heavy users of psychiatric services. Psychiatric Services. 1995; 46(12):1247-53. [DOI:10.1176/ps.46.12.1247] [PMID]

[77] Albert M, Becker T, Mccrone P, Thornicroft G. Social networks and mental health service utilization - a literature review. International Journal of Social Psychiatry. 1998; 44(4):248-66. [DOI:10.11 77/002076409804400402] [PMID]

[78] Barker G. Adolescents, social support and help-seeking behaviour: An international literature review and programme consultation with recommendations for action [Internet]. 2007 [Updated 2007]. Available from: https://apps.who.int/iris/ handle/10665/43778

[79] Li W, Dorstyn DS, Denson LA. Psychosocial correlates of college students' help-seeking intention: A meta-analysis. Professional Psychology: Research and Practice. 2014; 45(3):163-70 [DOI:10.1037/a0037118]

[80] Li W, Dorstyn DS, Denson LA. Predictors of mental health service use by young adults: A systematic review. Psychiatric Services. 2016; 67(9):946-56. [DOI:10.1176/appi.ps.201500280] [PMID] 
This Page Intentionally Left Blank 